\title{
Prediction of Supernova Rates in Known Galaxy-Galaxy Strong-lens Systems
}

DOI:

10.3847/1538-4357/aad5ea

\section{Document Version}

Final published version

Link to publication record in Manchester Research Explorer

\section{Citation for published version (APA):}

Shu, Y., Bolton, A. S., Mao, S., Kang, X., Li, G., \& Soraisam, M. (2018). Prediction of Supernova Rates in Known Galaxy-Galaxy Strong-lens Systems. The Astrophysical Journal, 864(1), 91. https://doi.org/10.3847/15384357/aad5ea

\section{Published in:}

The Astrophysical Journal

\section{Citing this paper}

Please note that where the full-text provided on Manchester Research Explorer is the Author Accepted Manuscript or Proof version this may differ from the final Published version. If citing, it is advised that you check and use the publisher's definitive version.

\section{General rights}

Copyright and moral rights for the publications made accessible in the Research Explorer are retained by the authors and/or other copyright owners and it is a condition of accessing publications that users recognise and abide by the legal requirements associated with these rights.

\section{Takedown policy}

If you believe that this document breaches copyright please refer to the University of Manchester's Takedown Procedures [http://man.ac.uk/04Y6Bo] or contact uml.scholarlycommunications@manchester.ac.uk providing relevant details, so we can investigate your claim.

\section{OPEN ACCESS}




\title{
Prediction of Supernova Rates in Known Galaxy-Galaxy Strong-lens Systems
}

\author{
Yiping Shu ${ }^{1,2,7}$, Adam S. Bolton ${ }^{3}$, Shude Mao ${ }^{4,5,6}$, Xi Kang ${ }^{1}$, Guoliang $\mathrm{Li}^{1}$, and Monika Soraisam ${ }^{3}$ \\ ${ }^{1}$ Purple Mountain Observatory, Chinese Academy of Sciences, 2 West Beijing Road, Nanjing 210008, People's Republic of China \\ ${ }^{2}$ Institute of Astronomy, University of Cambridge, Madingley Road, Cambridge CB3 0HA, UK; yiping.shu@pmo.ac.cn \\ ${ }^{3}$ National Optical Astronomy Observatory, 950 N. Cherry Avenue, Tucson, AZ 85719, USA; bolton@ noao.edu \\ ${ }^{4}$ Physics Department and Tsinghua Centre for Astrophysics, Tsinghua University, Beijing 100084, People's Republic of China \\ ${ }^{5}$ National Astronomical Observatories, Chinese Academy of Sciences, 20A Datun Road, Chaoyang District, Beijing 100012, People's Republic of China \\ ${ }^{6}$ Jodrell Bank Centre for Astrophysics, School of Physics and Astronomy, The University of Manchester, Oxford Road, Manchester M13 9PL, UK \\ Received 2018 January 22; revised 2018 June 24; accepted 2018 July 23; published 2018 September 4
}

\begin{abstract}
We propose a new strategy of finding strongly lensed supernovae (SNe) by monitoring known galaxy-scale stronglens systems. Strongly lensed SNe are potentially powerful tools for the study of cosmology, galaxy evolution, and stellar populations, but they are extremely rare. By targeting known strongly lensed star-forming galaxies, our strategy significantly boosts the detection efficiency for lensed SNe compared to a blind search. As a reference sample, we compile the 128 galaxy-galaxy strong-lens systems from the Sloan Lens ACS Survey (SLACS), the SLACS for the Masses Survey, and the Baryon Oscillation Spectroscopic Survey Emission-Line Lens Survey. Within this sample, we estimate the rates of strongly lensed Type Ia SN (SNIa) and core-collapse SN (CCSN) to be $1.23 \pm 0.12$ and $10.4 \pm 1.1$ events per year, respectively. The lensed SN images are expected to be widely separated with a median separation of 2 arcsec. Assuming a conservative fiducial lensing magnification factor of 5 for the most highly magnified SN image, we forecast that a monitoring program with a single-visit depth of $24.7 \mathrm{mag}$ ( $5 \sigma$ point source, $r$ band) and a cadence of 5 days can detect 0.49 strongly lensed SNIa event and 2.1 strongly lensed CCSN events per year within this sample. Our proposed targeted-search strategy is particularly useful for prompt and efficient identifications and follow-up observations of strongly lensed SN candidates. It also allows telescopes with small fields of view and limited time to efficiently discover strongly lensed SNe with a pencil-beam scanning strategy.
\end{abstract}

Key words: cosmology: observations - gravitational lensing: strong - supernovae: general

\section{Introduction}

Supernovae $(\mathrm{SNe})$ are powerful tools for understanding fundamental physics from cosmology to nucleosynthesis. Most prominently for cosmology, the "standard-candle" nature of Type Ia SN (SNIa) was used to discover the accelerating expansion of the universe (e.g., Riess et al. 1998; Schmidt et al. 1998; Perlmutter et al. 1999). By calibrating against distance-independent observables, Type II SNe have also been used for cosmological studies (e.g., Nugent et al. 2006; Jones et al. 2009; Kasen \& Woosley 2009; Poznanski et al. 2009; Olivares et al. 2010; de Jaeger et al. 2017), although the dispersion in the distance inferred from Type II SNe $(\sim 10 \%)$ is a factor of $\sim 2$ larger than that from SNe Ia (e.g., Betoule et al. 2014; Rubin \& Hayden 2016). Of particular interest for astrophysics, observations of the pre- or early explosion phases of an SN can constrain the properties of its progenitor, and by extension probe the physics of late-stage stellar evolution (e.g., Soderberg et al. 2008; Li et al. 2011a; Nugent et al. 2011; Dilday et al. 2012; Wang et al. 2013; McCully et al. 2014; Foley et al. 2015; Ganot et al. 2016; Tornambé et al. 2018).

The strong gravitational lensing effect furnishes another powerful tool for cosmology. From relative delays in the arrival times of multiple images, the Hubble constant can be measured for a given lensing mass model (e.g., Impey et al. 1998; Witt et al. 2000; Treu \& Koopmans 2002; Koopmans et al. 2003; Suyu et al. 2010, 2013, 2014; Bonvin et al. 2017; Wong et al. 2017). This so-called "time-delay cosmography" is independent of, and complementary to, other cosmological probes such

\footnotetext{
${ }^{7}$ Royal Society—K.C. Wong International Fellow.
}

as standard-candle SNIa, baryon acoustic oscillation (e.g., Eisenstein et al. 2005; Percival et al. 2010; Anderson et al. 2014; Alam et al. 2017; Wang et al. 2017; Zhao et al. 2017), and the fluctuation spectrum of the cosmic microwave background (e.g., Bennett et al. 2013; Planck Collaboration et al. 2016). Further observational development of the timedelay cosmography method is strongly motivated because the combination of different probes can mitigate the cosmological parameter degeneracies of each individual method. Furthermore, the lensed source is usually magnified by a factor of 10 or more, which makes studies of faint objects beyond the detection limit feasible (e.g., Bolton et al. 2006b; Quider et al. 2009; Christensen et al. 2012; Muzzin et al. 2012; Bussmann et al. 2013; Stark et al. 2015; Karman et al. 2016; Shu et al. 2016c; Spilker et al. 2016).

The combination of these two tools in the single phenomenon of strongly lensed $\mathrm{SNe}$ provides a simultaneous probe of cosmology and of SN physics. All but one time-delay cosmography measurement to-date have used the relatively more common phenomenon of strongly lensed quasi-stellar objects (QSOs). Compared to lensed QSOs, lensed SNe (and especially lensed SNIa) are more powerful for cosmological measurements because (1) the optical light curves from which time delays are determined are more regular and better understood for SNe (e.g., Barbon et al. 1979; Doggett \& Branch 1985; Kasen \& Woosley 2009; Sanders et al. 2015; Morozova et al. 2017) and (2) the intrinsic luminosity of some $\mathrm{SNe}$ can be inferred independently of lensing to constrain the absolute lensing magnifications. This extra constraint can be used to break known degeneracies in the lens modeling (e.g., Falco et al. 1985; Gorenstein et al. 1988; Saha 2000; 
Wucknitz 2002; Liesenborgs \& De Rijcke 2012; Schneider \& Sluse 2013, 2014) and lead to a more accurate measurement of the Hubble constant, provided the effects from microlensing can be corrected (e.g., Dobler \& Keeton 2006; Yahalomi et al. 2017; Foxley-Marrable et al. 2018; Goldstein et al. 2018). Furthermore, time delays between lensed images make strong gravitational lensing a natural time machine. The typical time delays in galaxy-scale strong lenses are on the order of 1 day to 100 days. One can thus monitor the SN explosion at the predicted location and time after detecting the leading lensed image, and catch the trailing images early in the explosive phase. It is in fact possible to watch the same explosion multiple times if there are more than two lensed images (e.g., Kelly et al. 2015, 2016). A significant sample of strongly lensed SNe could therefore provide significant improvement in our understanding of pre- and early explosion progenitor properties, especially in combination with the lensing magnification effect.

Despite the great power of strongly lensed SN systems, there have been only three serendipitous discoveries to date (Quimby et al. 2014; Kelly et al. 2015; Goobar et al. 2017). The difficulty comes from the fact that a strongly lensed $\mathrm{SN}$ system is an $\mathcal{O}$ $\left(\mathrm{rare}^{3}\right)$ event. Strong-lensing events (i.e., with multiple lensed images) are rare because they require a close alignment between the observer, the lens, and the source. Studies suggest that the strong-lens event rate on galaxy scales is on the order of 1 in 1000 (e.g., Bolton et al. 2006a; Marshall et al. 2009). At the same time, $\mathrm{SN}$ events are rare too. The SN rate in starburst galaxies is found to be a few to 10 events per century (e.g., Tammann et al. 1994; Richmond et al. 1998; Mannucci et al. 2003; Diehl et al. 2006; Adams et al. 2013). Finally, SNe fade after explosion on restframe timescales of $\sim 50$ days (e.g., Richardson et al. $2002 ; \mathrm{Li}$ et al. 2011c). Therefore, observations need to be made in the window before the $\mathrm{SNe}$ go below the detection limits.

A large sample of strongly lensed $\mathrm{SNe}$ is expected to be discovered by the upcoming Large Synoptic Survey Telescope (LSST) project. Oguri \& Marshall (2010) predict that LSST will discover 46 strongly lensed SNIa and 84 strongly lensed core-collapse SN (CCSN, including Type $\mathrm{Ib} / \mathrm{c}$ and Type II) events with image separations larger than $0 . " 5$ in 10 years. Kostrzewa-Rutkowska et al. (2013) and Goldstein \& Nugent (2017) predict furthermore that LSST will detect 220-1400 strongly lensed SNIa and 3800 strongly lensed CCSN events with image separations as small as 0 !" 1 in 10 years. The numbers of strongly lensed $\mathrm{SNe}$ in these latter predictions become similar to the prediction of Oguri \& Marshall (2010) if limited to events with image separations larger than $0 . " 5$ and magnifications solely due to brightest images. Further limiting to median image separations larger than 2", Goldstein \& Nugent (2017) predict that LSST will detect an average of five (this number is estimated from their Figure 5 as $\sim 20 \%-25 \%$ of the total predictions) strongly lensed SNIa events per year.

In this paper, we propose a new strategy of finding strongly lensed SNe through monitoring of a sample of known galaxyscale strong-lens systems. The advantages of this strategy are three-fold. First, any SN occurring at the location of a known strongly lensed star-forming galaxy has a significantly higher probability of being strongly lensed and multiply imaged, as compared to an SN detected in the field. Second, the multiple SN images detected in this sample will have the same relatively wide image separations as their lensed host galaxies (median of $2^{\prime \prime}$ ), facilitating intensive early time observation of trailing images and precise measurements of time delays. Lastly, compared to galaxy clusters behind which previous searches for lensed $\mathrm{SNe}$ were carried out (e.g. Goobar et al. 2009; Postman et al. 2012; Pan \& Lobe 2013; Rodney et al. 2015; Petrushevska et al. 2016, 2018), the foreground lens objects of our work as primarily isolated early-type galaxies, which have relatively smooth and simple mass profiles that can be more robustly constrained from lensing data when compared to galaxy groups or clusters. This advantage is significant for the application of detected strongly lensed SNe to time-delay cosmography.

This paper is organized as follows. Section 2 introduces the compilation of strong-lens systems used. Section 3 describes our procedure for estimating the star formation rates and $\mathrm{SN}$ rates in the lensed sources. Predictions for detectable SN rates given particular survey depths are presented in Section 4. Discussions and conclusions are given in Sections 5 and 6. Throughout the paper, we adopt a fiducial cosmological model with $\Omega_{\mathrm{m}}=0.308, \Omega_{\Lambda}=0.692$, and $H_{0}=67.8 \mathrm{~km} \mathrm{~s}^{-1} \mathrm{Mpc}^{-1}$ (Planck Collaboration et al. 2016).

\section{Strong-lens Sample Compilation}

To date, almost 300 galaxy-scale strong-lens systems have been discovered (e.g., Walsh et al. 1979; Muñoz et al. 1998; Browne et al. 2003; Bolton et al. 2008; Faure et al. 2008; Treu et al. 2011; Brownstein et al. 2012; Inada et al. 2012; More et al. 2012, 2016; Sonnenfeld et al. 2013; Stark et al. 2013; Vieira et al. 2013; Pawase et al. 2014; Shu et al. 2016b, 2017; Negrello et al. 2017; Sonnenfeld et al. 2018). In this work, we build a compilation of 128 strong-lens systems from the Sloan Lens ACS Survey (SLACS; Bolton et al. 2008, 63 systems), the SLACS for the Masses Survey (S4TM; Shu et al. 2017, 40 systems), and the Baryon Oscillation Spectroscopy Survey (BOSS) Emission-Line Lens Survey (BELLS; Brownstein et al. 2012, 25 systems). The strong-lens systems in these three surveys are selected by the technique introduced in Bolton et al. (2004), which ensures that the lensing object is a massive early-type galaxy while the lensed object is a galaxy with significant star formation as indicated by the securely detected [O II] $\lambda \lambda 3727$ emission along with (in many cases) $\mathrm{H} \beta$, [O III] 4959, [O III] 5007 , and $\mathrm{H} \alpha$ emission. Accurate lens models have been derived from Hubble Space Telescope (HST) imaging data for all the systems. The lens and source redshifts are spectroscopically determined. The median lens and source redshifts for the SLACS, S4TM, and BELLS lens samples are $\left(z_{L}=0.20, z_{S}=0.60\right),\left(z_{L}=0.16, z_{S}=0.59\right)$, and $\left(z_{L}=0.52\right.$, $z_{S}=1.18$ ), respectively. The median image separation in this compilation is $2^{\prime \prime}$.

\section{Star Formation Rate and Supernova Rates}

The rate of core-collapse SN (CCSN, including Type $\mathrm{Ib} / \mathrm{c}$ and Type II) in a galaxy, associated with the final collapse of short-lived massive stars, is significantly correlated with the star formation rate (SFR; e.g., Madau et al. 1998; Dahlén \& Fransson 1999; Hopkins \& Beacom 2006; Dahlen et al. 2012; Melinder et al. 2012). The rate of SNIa depends on other hostgalaxy properties in addition to SFR (e.g., Sullivan et al. 2006; Li et al. 2011b; Smith et al. 2012; Graur et al. 2015). Following previous work (e.g., Greggio et al. 2008; Maoz et al. 2011), we determine the SNIa rate in our sample by convolving the star formation history (SFH) with the SNIa delay-time distribution, which has been calibrated against observations to account for some of the most important host-galaxy property dependences (for instance, on host-galaxy mass and age.) 
Table 1

Useful Properties of the Compilation of Strong Lenses

\begin{tabular}{|c|c|c|c|c|c|c|c|c|c|}
\hline Target & Position & $z_{L}$ & (4) & (5) & $\begin{array}{l}f_{\text {fiber }} \\
(6)\end{array}$ & $\begin{array}{c}F_{[\mathrm{O} \text { II }]} \\
(7)\end{array}$ & $\begin{array}{c}\mathrm{SFR} \\
M_{\odot} \mathrm{yr}^{-1} \\
(8)\end{array}$ & $\begin{array}{c}N_{\text {cc }} \\
\mathrm{yr}^{-1} \\
(9)\end{array}$ & $\begin{array}{c}N_{\text {Ia }} \\
\mathrm{yr}^{-1} \\
(10)\end{array}$ \\
\hline SLACSJ 0008-0004 & 00:08:02.96-00:04:08.20 & 0.44 & 1.19 & 5 & $43 \%$ & $\ldots$ & $\ldots$ & $\ldots$ & $\ldots$ \\
\hline SLACSJ 0029-0055 & $00: 29: 07.77-00: 55: 50.50$ & 0.23 & 0.93 & 23 & $61 \%$ & $3.3 \pm 1.1$ & $5.3 \pm 3.0$ & $0.019 \pm 0.011$ & $0.0023 \pm 0.0013$ \\
\hline SLACSJ 0037-0942 & $00: 37: 53.22-09: 42: 20.14$ & 0.20 & 0.63 & 6 & $34 \%$ & $2.2 \pm 0.5$ & $6.3 \pm 3.2$ & $0.027 \pm 0.014$ & $0.0035 \pm 0.0018$ \\
\hline SLACSJ $0044+0113^{*}$ & $00: 44: 02.90+01: 13: 12.50$ & 0.12 & 0.20 & 2 & $53 \%$ & $14.5 \pm 2.4$ & $2.5 \pm 1.2$ & $0.014 \pm 0.007$ & $0.0022 \pm 0.0010$ \\
\hline SLACSJ 0109+1500 & $01: 09: 33.74+15: 00: 32.50$ & 0.29 & 0.52 & 2 & $55 \%$ & $2.4 \pm 1.0$ & $6.1 \pm 3.8$ & $0.028 \pm 0.017$ & $0.0038 \pm 0.0023$ \\
\hline SLACSJ 0157-0056 & $01: 57: 58.94-00: 56: 26.10$ & 0.51 & 0.92 & 2 & $28 \%$ & $2.5 \pm 1.4$ & $83.9 \pm 60.2$ & $0.305 \pm 0.219$ & $0.0368 \pm 0.0264$ \\
\hline SLACSJ 0216-0813 & $02: 16: 52.53-08: 13: 45.44$ & 0.33 & 0.52 & 3 & $26 \%$ & $4.6 \pm 1.1$ & $19.6 \pm 10.1$ & $0.090 \pm 0.046$ & $0.0121 \pm 0.0062$ \\
\hline SLACSJ $0252+0039$ & $02: 52: 45.21+00: 39: 58.40$ & 0.28 & 0.98 & 16 & $53 \%$ & $6.3 \pm 0.6$ & $19.2 \pm 8.8$ & $0.068 \pm 0.031$ & $0.0080 \pm 0.0037$ \\
\hline SLACSJ 0330-0020 & $03: 30: 12.14-00: 20: 51.90$ & 0.35 & 1.07 & 4 & $47 \%$ & $4.7 \pm 0.9$ & $98.3 \pm 47.8$ & $0.332 \pm 0.162$ & $0.0387 \pm 0.0188$ \\
\hline SLACSJ 0405-0455 & 04:05:35.42-04:55:52.40 & 0.08 & 0.81 & 16 & $73 \%$ & $1.7 \pm 0.3$ & $2.1 \pm 1.0$ & $0.008 \pm 0.004$ & $0.0010 \pm 0.0005$ \\
\hline SLACSJ $0728+3835$ & $07: 28: 04.95+38: 35: 25.70$ & 0.21 & 0.69 & 10 & $52 \%$ & $1.8 \pm 0.9$ & $2.8 \pm 1.9$ & $0.012 \pm 0.008$ & $0.0015 \pm 0.0010$ \\
\hline SLACSJ $0737+3216$ & $07: 37: 28.44+32: 16: 18.66$ & 0.32 & 0.58 & 14 & $52 \%$ & $7.2 \pm 1.5$ & $4.6 \pm 2.3$ & $0.020 \pm 0.010$ & $0.0027 \pm 0.0013$ \\
\hline SLACSJ $0822+2652$ & $08: 22: 42.32+26: 52: 43.50$ & 0.24 & 0.59 & 7 & $46 \%$ & $2.1 \pm 0.5$ & $3.2 \pm 1.6$ & $0.014 \pm 0.007$ & $0.0018 \pm 0.0009$ \\
\hline SLACSJ $0841+3824$ & $08: 41: 28.81+38: 24: 13.70$ & 0.12 & 0.66 & 5 & $30 \%$ & $\ldots$ & $\ldots$ & $\ldots$ & $\ldots$ \\
\hline SLACSJ $0903+4116$ & 09:03:15.19+41:16:09.10 & 0.43 & 1.06 & 8 & $48 \%$ & $3.1 \pm 1.9$ & $26.9 \pm 20.7$ & $0.091 \pm 0.070$ & $0.0106 \pm 0.0082$ \\
\hline SLACSJ 0912+0029* & 09:12:05.31+00:29:01.18 & 0.16 & 0.32 & 6 & $26 \%$ & $4.4 \pm 2.0$ & $2.5 \pm 1.6$ & $0.013 \pm 0.008$ & $0.0019 \pm 0.0012$ \\
\hline SLACSJ 0935-0003 & $09: 35: 43.93-00: 03: 34.80$ & 0.35 & 0.47 & 3 & $34 \%$ & $3.2 \pm 2.7$ & $6.9 \pm 6.6$ & $0.033 \pm 0.031$ & $0.0045 \pm 0.0043$ \\
\hline SLACSJ 0936+0913 & 09:36:00.77+09:13:35.80 & 0.19 & 0.59 & 7 & $55 \%$ & $2.4 \pm 0.7$ & $3.0 \pm 1.6$ & $0.013 \pm 0.007$ & $0.0018 \pm 0.0010$ \\
\hline SLACSJ 0946+1006 & $09: 46: 56.68+10: 06: 52.80$ & 0.22 & 0.61 & 18 & $46 \%$ & $7.0 \pm 1.9$ & $4.6 \pm 2.4$ & $0.020 \pm 0.011$ & $0.0026 \pm 0.0014$ \\
\hline SLACSJ $0955+0101^{*}$ & $09: 55: 19.72+01: 01: 44.40$ & 0.11 & 0.32 & 4 & $50 \%$ & $5.2 \pm 0.9$ & $2.1 \pm 1.0$ & $0.011 \pm 0.005$ & $0.0016 \pm 0.0008$ \\
\hline SLACSJ $0956+5100$ & $09: 56: 29.78+51: 00: 06.39$ & 0.24 & 0.47 & 8 & $45 \%$ & $4.6 \pm 1.4$ & $2.9 \pm 1.6$ & $0.014 \pm 0.007$ & $0.0019 \pm 0.0010$ \\
\hline SLACSJ 0959+0410 & 09:59:44.07+04:10:17.00 & 0.13 & 0.54 & 5 & $56 \%$ & $3.9 \pm 1.7$ & $4.9 \pm 3.1$ & $0.023 \pm 0.014$ & $0.0030 \pm 0.0019$ \\
\hline SLACSJ 0959+4416 & 09:59:00.96+44:16:39.40 & 0.24 & 0.53 & 9 & $57 \%$ & $2.6 \pm 0.7$ & $1.9 \pm 1.0$ & $0.009 \pm 0.005$ & $0.0011 \pm 0.0006$ \\
\hline SLACSJ 1016+3859 & $10: 16: 22.86+38: 59: 03.30$ & 0.17 & 0.44 & 5 & $40 \%$ & $1.8 \pm 0.7$ & $1.6 \pm 1.0$ & $0.008 \pm 0.005$ & $0.0010 \pm 0.0006$ \\
\hline SLACSJ $1020+1122$ & $10: 20: 26.54+11: 22: 41.10$ & 0.28 & 0.55 & 6 & $51 \%$ & $2.6 \pm 1.0$ & $3.3 \pm 1.9$ & $0.015 \pm 0.009$ & $0.0019 \pm 0.0012$ \\
\hline SLACSJ $1023+4230$ & $10: 23: 32.26+42: 30: 01.80$ & 0.19 & 0.70 & 11 & $39 \%$ & $\ldots$ & $\ldots$ & $\ldots$ & $\ldots$ \\
\hline SLACSJ $1029+0420$ & $10: 29: 22.94+04: 20: 01.80$ & 0.10 & 0.62 & 4 & $47 \%$ & $2.3 \pm 0.8$ & $7.6 \pm 4.3$ & $0.033 \pm 0.019$ & $0.0043 \pm 0.0024$ \\
\hline SLACSJ $1032+5322^{*}$ & $10: 32: 35.84+53: 22: 34.90$ & 0.13 & 0.33 & 3 & $38 \%$ & $9.2 \pm 1.0$ & $6.0 \pm 2.8$ & $0.032 \pm 0.015$ & $0.0045 \pm 0.0021$ \\
\hline SLACSJ $1100+5329$ & $11: 00: 24.39+53: 29: 13.71$ & 0.32 & 0.86 & 13 & $28 \%$ & $6.8 \pm 2.0$ & $30.7 \pm 16.5$ & $0.116 \pm 0.062$ & $0.0142 \pm 0.0076$ \\
\hline SLACSJ $1103+5322$ & $11: 03: 08.21+53: 22: 28.20$ & 0.16 & 0.74 & 3 & $24 \%$ & $5.4 \pm 3.1$ & $76.3 \pm 55.5$ & $0.308 \pm 0.224$ & $0.0388 \pm 0.0283$ \\
\hline SLACSJ $1106+5228$ & $11: 06: 46.16+52: 28: 37.70$ & 0.10 & 0.41 & 25 & $23 \%$ & $8.3 \pm 3.5$ & $2.3 \pm 1.4$ & $0.011 \pm 0.007$ & $0.0016 \pm 0.0010$ \\
\hline SLACSJ $1112+0826$ & $11: 12: 50.60+08: 26: 10.40$ & 0.27 & 0.63 & 4 & $11 \%$ & $2.6 \pm 0.8$ & $32.0 \pm 17.6$ & $0.137 \pm 0.075$ & $0.0179 \pm 0.0098$ \\
\hline SLACSJ $1134+6027$ & $11: 34: 05.88+60: 27: 13.20$ & 0.15 & 0.47 & 2 & $18 \%$ & $\ldots$ & $\ldots$ & $\ldots$ & $\ldots$ \\
\hline SLACSJ $1142+1001$ & $11: 42: 57.35+10: 01: 11.80$ & 0.22 & 0.50 & 3 & $42 \%$ & $2.5 \pm 0.7$ & $5.1 \pm 2.7$ & $0.024 \pm 0.012$ & $0.0032 \pm 0.0017$ \\
\hline SLACSJ $1143-0144^{*}$ & $11: 43: 29.64-01: 44: 30.00$ & 0.11 & 0.40 & 4 & $18 \%$ & $9.0 \pm 1.1$ & $16.6 \pm 7.7$ & $0.083 \pm 0.039$ & $0.0116 \pm 0.0054$ \\
\hline SLACSJ $1153+4612$ & $11: 53: 10.79+46: 12: 05.30$ & 0.18 & 0.88 & 10 & $59 \%$ & $2.7 \pm 0.7$ & $7.8 \pm 4.0$ & $0.029 \pm 0.015$ & $0.0035 \pm 0.0018$ \\
\hline SLACSJ $1204+0358$ & $12: 04: 44.07+03: 58: 06.39$ & 0.16 & 0.63 & 9 & $49 \%$ & $4.9 \pm 1.4$ & $6.9 \pm 3.7$ & $0.030 \pm 0.016$ & $0.0039 \pm 0.0021$ \\
\hline SLACSJ $1205+4910$ & $12: 05: 40.44+49: 10: 29.38$ & 0.21 & 0.48 & 13 & $46 \%$ & $6.2 \pm 0.6$ & $2.7 \pm 1.2$ & $0.013 \pm 0.006$ & $0.0017 \pm 0.0008$ \\
\hline SLACSJ $1213+6708$ & $12: 13: 40.58+67: 08: 29.00$ & 0.12 & 0.64 & 8 & $32 \%$ & $2.8 \pm 0.8$ & $7.4 \pm 3.9$ & $0.032 \pm 0.017$ & $0.0041 \pm 0.0022$ \\
\hline SLACSJ $1218+0830$ & $12: 18: 26.70+08: 30: 50.30$ & 0.14 & 0.72 & 4 & $30 \%$ & $\ldots$ & $\ldots$ & $\ldots$ & $\ldots$ \\
\hline SLACSJ $1250+0523$ & $12: 50: 28.26+05: 23: 49.07$ & 0.23 & 0.80 & 10 & $46 \%$ & $6.2 \pm 1.7$ & $17.6 \pm c$ & $0.069 \pm 0$ & $0.0086 \pm 0.0045$ \\
\hline SLACSJ $1251-0208$ & $12: 51: 35.71-02: 08: 05.17$ & 0.22 & 0.78 & 4 & $48 \%$ & $3.4 \pm 1.0$ & $20.4 \pm 11.0$ & $0.080 \pm 0.043$ & $0.0100 \pm 0.0054$ \\
\hline SLACSJ $1402+6321$ & $14: 02: 28.22+63: 21: 33.34$ & 0.20 & 0.48 & 33 & $37 \%$ & $\ldots$ & $\ldots$ & $\ldots$ & $\ldots$ \\
\hline SLACSJ $1403+0006$ & $14: 03: 29.49+00: 06: 41.30$ & 0.19 & 0.47 & 3 & $45 \%$ & $\ldots$ & $\cdots$ & $\cdots$ & $\cdots$ \\
\hline SLACSJ $1416+5136$ & $14: 16: 22.34+51: 36: 30.40$ & 0.30 & 0.81 & 4 & $22 \%$ & $3.1 \pm 1.4$ & $54.0 \pm 34.7$ & $0.209 \pm 0.134$ & $0.0259 \pm 0.0166$ \\
\hline SLACSJ $1420+6019$ & $14: 20: 15.85+60: 19: 14.80$ & 0.06 & 0.54 & 13 & $49 \%$ & $7.0 \pm 1.5$ & $3.9 \pm 2.0$ & $0.018 \pm 0.009$ & $0.0024 \pm 0.0012$ \\
\hline SLACSJ $1430+4105$ & $14: 30: 04.10+41: 05: 57.10$ & 0.28 & 0.58 & 6 & $35 \%$ & $8.6 \pm 1.8$ & $18.5 \pm 9.2$ & $0.082 \pm 0.041$ & $0.0109 \pm 0.0054$ \\
\hline SLACSJ $1432+6317$ & $14: 32: 13.34+63: 17: 03.80$ & 0.12 & 0.66 & 4 & $38 \%$ & $9.2 \pm 1.4$ & $45.3 \pm 21.5$ & $0.190 \pm 0.090$ & $0.0245 \pm 0.0116$ \\
\hline SLACSJ $1436-0000$ & $14: 36: 27.54-00: 00: 29.10$ & 0.29 & 0.80 & 4 & $43 \%$ & $2.7 \pm 0.8$ & $18.9 \pm 10.0$ & $0.073 \pm 0.039$ & $0.0091 \pm 0.0048$ \\
\hline SLACSJ $1443+0304$ & $14: 43: 19.62+03: 04: 08.25$ & 0.13 & 0.42 & 7 & $41 \%$ & $1.5 \pm 0.5$ & $0.9 \pm 0.5$ & $0.005 \pm 0.003$ & $0.0006 \pm 0.0003$ \\
\hline SLACSJ $1451-0239$ & $14: 51: 28.19-02: 39: 36.40$ & 0.13 & 0.52 & 11 & $45 \%$ & $5.3 \pm 1.1$ & $3.3 \pm 1.7$ & $0.015 \pm 0.008$ & $0.0021 \pm 0.0010$ \\
\hline SLACSJ $1525+3327$ & $15: 25: 06.70+33: 27: 47.40$ & 0.36 & 0.72 & 4 & $32 \%$ & $3.7 \pm 0.7$ & $23.6 \pm 11.4$ & $0.096 \pm 0.046$ & $0.0122 \pm 0.0059$ \\
\hline SLACSJ $1531-0105$ & $15: 31: 50.08-01: 05: 45.60$ & 0.16 & 0.74 & 10 & $28 \%$ & $\ldots$ & $\ldots$ & $\ldots$ & $\ldots$ \\
\hline SLACSJ $1538+5817$ & $15: 38: 12.94+58: 17: 09.69$ & 0.14 & 0.53 & 8 & $41 \%$ & $15.0 \pm 1.4$ & $16.5 \pm 7.5$ & $0.075 \pm 0.035$ & $0.0101 \pm 0.0046$ \\
\hline SLACSJ $1621+3931$ & $16: 21: 32.99+39: 31: 44.60$ & 0.24 & 0.60 & 8 & $47 \%$ & $9.7 \pm 8.0$ & $13.5 \pm 12.6$ & $0.059 \pm 0.055$ & $0.0077 \pm 0.0072$ \\
\hline SLACSJ $1627-0053$ & $16: 27: 46.45-00: 53: 57.56$ & 0.21 & 0.52 & 20 & $49 \%$ & $3.7 \pm 0.7$ & $1.3 \pm 0.6$ & $0.006 \pm 0.003$ & $0.0008 \pm 0.0004$ \\
\hline SLACSJ $1630+4520$ & $16: 30: 28.16+45: 20: 36.28$ & 0.25 & 0.79 & 9 & $22 \%$ & $2.7 \pm 1.0$ & $16.7 \pm 9.6$ & $0.065 \pm 0.038$ & $0.0081 \pm 0.0047$ \\
\hline SLACSJ $1636+4707$ & $16: 36: 02.62+47: 07: 29.57$ & 0.23 & 0.67 & 8 & $62 \%$ & $1.5 \pm 0.6$ & $2.3 \pm 1.3$ & $0.010 \pm 0.006$ & $0.0012 \pm 0.0007$ \\
\hline SLACSJ $2238-0754$ & $22: 38: 40.20-07: 54: 56.05$ & 0.14 & 0.71 & 12 & $52 \%$ & $5.3 \pm 1.3$ & $7.4 \pm 3.8$ & $0.030 \pm 0.016$ & $0.0039 \pm 0.0020$ \\
\hline SLACSJ $2300+0022$ & $23: 00: 53.14+00: 22: 37.95$ & 0.23 & 0.46 & 12 & $49 \%$ & $2.0 \pm 0.8$ & $0.7 \pm 0.5$ & $0.004 \pm 0.002$ & $0.0005 \pm 0.0003$ \\
\hline SLACSJ $2303+1422$ & $23: 03: 21.72+14: 22: 17.91$ & 0.16 & 0.52 & 8 & $41 \%$ & $1.6 \pm 0.8$ & $1.6 \pm 1.1$ & $0.007 \pm 0.005$ & $0.0010 \pm 0.0007$ \\
\hline SLACSJ 2321-0939 & $23: 21: 20.93-09: 39: 10.27$ & 0.08 & 0.53 & 12 & $33 \%$ & $7.6 \pm 1.6$ & $6.7 \pm 3.3$ & $0.031 \pm 0.015$ & $0.0041 \pm 0.0020$ \\
\hline
\end{tabular}


Table 1

(Continued)

\begin{tabular}{|c|c|c|c|c|c|c|c|c|c|}
\hline Target & Position & (3) & (4) & (5) & $\begin{array}{l}f_{\text {fiber }} \\
(6)\end{array}$ & $\begin{array}{c}\left.F_{[\mathrm{O}} \mathrm{II}\right] \\
(7)\end{array}$ & $\begin{array}{c}\mathrm{SFR} \\
M_{\odot} \mathrm{yr}^{-1} \\
(8)\end{array}$ & $\begin{array}{c}N_{\mathrm{cc}} \\
\mathrm{yr}^{-1} \\
(9)\end{array}$ & $\begin{array}{c}N_{\text {Ia }} \\
\mathrm{yr}^{-1} \\
(10)\end{array}$ \\
\hline $\begin{array}{l}\text { SLACSJ } 2341+0000 \\
\text { Target }\end{array}$ & $\begin{array}{c}23: 41: 11.57+00: 00: 18.70 \\
\text { Position }\end{array}$ & $\begin{array}{l}0.19 \\
z_{L}\end{array}$ & $\begin{array}{l}0.81 \\
z_{S}\end{array}$ & $\begin{array}{c}11 \\
\mu\end{array}$ & $\begin{array}{l}44 \% \\
f_{\text {fiber }}\end{array}$ & $F_{[\mathrm{O} \text { I] }}$ & $\begin{array}{r}\cdots \\
\mathrm{SFR}^{\cdots} \\
M_{\odot} \mathrm{yr}^{-1} \\
(8)\end{array}$ & $\begin{array}{c}N_{\mathrm{cc}} \\
\mathrm{yr}^{-1} \\
(9)\end{array}$ & $\begin{array}{c}N^{\cdots} \\
N_{\text {Ia }} \\
\mathrm{yr}^{-1} \\
(10)\end{array}$ \\
\hline S4TMJ 0143-1006 & $01: 43: 56.58-10: 06: 33.70$ & 0.22 & 1.10 & 3 & $33 \%$ & $4.3 \pm 1.8$ & $159.5 \pm 99.1$ & $0.531 \pm 0.330$ & $0.0614 \pm 0.0381$ \\
\hline S4TMJ 0159-0006 & $01: 59: 30.14-00: 06: 12.40$ & 0.16 & 0.75 & 6 & $56 \%$ & $1.7 \pm 0.6$ & $5.2 \pm 2.9$ & $0.021 \pm 0.012$ & $0.0026 \pm 0.0015$ \\
\hline S4TMJ 0324+0045 & $03: 24: 15.50+00: 45: 05.50$ & 0.32 & 0.92 & 14 & $77 \%$ & $1.5 \pm 0.7$ & $2.9 \pm 1.8$ & $0.010 \pm 0.007$ & $0.0013 \pm 0.0008$ \\
\hline S4TMJ 0324-0110 & 03:24:54.50-01:10:29.10 & 0.45 & 0.62 & 4 & $79 \%$ & $2.1 \pm 0.4$ & $3.5 \pm 1.7$ & $0.015 \pm 0.008$ & $0.0020 \pm 0.0010$ \\
\hline S4TMJ $0753+3416$ & $07: 53: 46.21+34: 16: 33.60$ & 0.14 & 0.96 & 27 & $56 \%$ & $6.7 \pm 2.3$ & $11.2 \pm 6.3$ & $0.040 \pm 0.023$ & $0.0048 \pm 0.0027$ \\
\hline S4TMJ 0754+1927 & $07: 54: 28.52+19: 27: 28.10$ & 0.15 & 0.74 & 5 & $43 \%$ & $4.1 \pm 1.7$ & $17.9 \pm 10.9$ & $0.072 \pm 0.044$ & $0.0091 \pm 0.0055$ \\
\hline S4TMJ $0757+1956$ & $07: 57: 48.99+19: 56: 16.30$ & 0.12 & 0.83 & 9 & $32 \%$ & $5.3 \pm 1.4$ & $28.4 \pm 15.0$ & $0.108 \pm 0.057$ & $0.0134 \pm 0.0070$ \\
\hline S4TMJ $0826+5630$ & $08: 26: 39.86+56: 30: 35.99$ & 0.13 & 1.29 & 85 & $61 \%$ & $\ldots$ & $\ldots$ & $\ldots$ & $\ldots$ \\
\hline S4TMJ $0847+2348$ & $08: 47: 27.69+23: 48: 19.50$ & 0.16 & 0.53 & 17 & $79 \%$ & $3.1 \pm 0.7$ & $0.8 \pm 0.4$ & $0.004 \pm 0.002$ & $0.0005 \pm 0.0003$ \\
\hline S4TMJ $0851+0505$ & $08: 51: 41.89+05: 05: 07.00$ & 0.13 & 0.64 & 6 & $69 \%$ & $5.9 \pm 1.8$ & $8.3 \pm 4.5$ & $0.036 \pm 0.019$ & $0.0046 \pm 0.0025$ \\
\hline S4TMJ 0920+3028 & $09: 20: 48.28+30: 28: 18.40$ & 0.29 & 0.39 & 8 & $48 \%$ & $3.3 \pm 0.5$ & $1.1 \pm 0.5$ & $0.006 \pm 0.003$ & $0.0008 \pm 0.0004$ \\
\hline S4TMJ 0955+3014 & $09: 55: 57.49+30: 14: 50.90$ & 0.32 & 0.47 & 4 & $72 \%$ & $2.4 \pm 2.2$ & $1.8 \pm 1.8$ & $0.009 \pm 0.009$ & $0.0012 \pm 0.0012$ \\
\hline S4TMJ 0956+5539 & $09: 56: 54.84+55: 39: 47.30$ & 0.20 & 0.85 & 19 & $46 \%$ & $\ldots$ & $\ldots$ & $\ldots$ & $\ldots$ \\
\hline S4TMJ $1010+3124$ & $10: 10: 26.80+31: 24: 17.60$ & 0.17 & 0.42 & 4 & $48 \%$ & $5.0 \pm 1.2$ & $4.9 \pm 2.5$ & $0.024 \pm 0.012$ & $0.0033 \pm 0.0017$ \\
\hline S4TMJ $1031+3026$ & $10: 31: 21.01+30: 26: 58.00$ & 0.17 & 0.75 & 5 & $69 \%$ & $7.1 \pm 1.9$ & $20.0 \pm 10.4$ & $0.080 \pm 0.042$ & $0.0101 \pm 0.0053$ \\
\hline S4TMJ $1040+3626^{*}$ & $10: 40: 58.50+36: 26: 28.60$ & 0.12 & 0.28 & 3 & $78 \%$ & $3.4 \pm 0.7$ & $0.8 \pm 0.4$ & $0.004 \pm 0.002$ & $0.0006 \pm 0.0003$ \\
\hline S4TMJ $1041+0112^{*}$ & $10: 41: 22.85+01: 12: 24.20$ & 0.10 & 0.22 & 5 & $73 \%$ & $4.2 \pm 1.2$ & $0.3 \pm 0.2$ & $0.002 \pm 0.001$ & $0.0002 \pm 0.0001$ \\
\hline S4TMJ $1048+1313$ & $10: 48: 09.40+13: 13: 52.90$ & 0.13 & 0.67 & 4 & $45 \%$ & $4.2 \pm 3.7$ & $18.3 \pm 18.2$ & $0.077 \pm 0.076$ & $0.0099 \pm 0.0098$ \\
\hline S4TMJ $1051+4439$ & $10: 51: 09.41+44: 39: 08.51$ & 0.16 & 0.54 & 3 & $52 \%$ & $8.0 \pm 2.0$ & $17.3 \pm 8.8$ & $0.079 \pm 0.040$ & $0.0105 \pm 0.0054$ \\
\hline S4TMJ $1056+4141$ & $10: 56: 57.61+41: 41: 14.60$ & 0.13 & 0.83 & 10 & $76 \%$ & $3.2 \pm 0.5$ & $6.2 \pm 2.9$ & $0.024 \pm 0.011$ & $0.0029 \pm 0.0014$ \\
\hline S4TMJ $1101+1523$ & $11: 01: 13.13+15: 23: 39.60$ & 0.18 & 0.52 & 5 & $48 \%$ & $2.7 \pm 0.8$ & $3.5 \pm 1.9$ & $0.016 \pm 0.009$ & $0.0022 \pm 0.0012$ \\
\hline S4TMJ $1116+0729$ & $11: 16: 41.66+07: 29: 45.60$ & 0.17 & 0.69 & 4 & $17 \%$ & $\cdots$ & $\cdots$ & $\cdots$ & $\cdots$ \\
\hline S4TMJ $1127+2312^{*}$ & $11: 27: 38.70+23: 12: 44.40$ & 0.13 & 0.36 & 8 & $19 \%$ & $5.7 \pm 0.8$ & $4.2 \pm 2.0$ & $0.022 \pm 0.010$ & $0.0030 \pm 0.0014$ \\
\hline S4TMJ $1137+1818$ & $11: 37: 28.61+18: 18: 12.40$ & 0.12 & 0.46 & 10 & $23 \%$ & $3.5 \pm 0.7$ & $3.7 \pm 1.8$ & $0.018 \pm 0.009$ & $0.0024 \pm 0.0012$ \\
\hline S4TMJ $1142+2509$ & $11: 42: 38.23+25: 09: 05.50$ & 0.16 & 0.66 & 18 & $83 \%$ & $3.4 \pm 0.9$ & $1.6 \pm 0.8$ & $0.007 \pm 0.003$ & $0.0009 \pm 0.0004$ \\
\hline S4TMJ $1144+0436^{*}$ & $11: 44: 40.13+04: 36: 50.50$ & 0.10 & 0.26 & 5 & $62 \%$ & $10.5 \pm 2.2$ & $1.5 \pm 0.8$ & $0.008 \pm 0.004$ & $0.0012 \pm 0.0006$ \\
\hline S4TMJ $1213+2930$ & $12: 13: 03.72+29: 30: 22.40$ & 0.09 & 0.60 & 21 & $49 \%$ & $4.2 \pm 1.3$ & $2.0 \pm 1.1$ & $0.009 \pm 0.005$ & $0.0011 \pm 0.0006$ \\
\hline S4TMJ $1301+0834$ & $13: 01: 26.88+08: 34: 25.20$ & 0.09 & 0.53 & 9 & $64 \%$ & $8.9 \pm 6.5$ & $5.8 \pm 5.0$ & $0.026 \pm 0.023$ & $0.0035 \pm 0.0030$ \\
\hline S4TMJ $1330+1750^{*}$ & $13: 30: 31.40+17: 50: 40.50$ & 0.21 & 0.37 & 4 & $50 \%$ & $2.9 \pm 0.6$ & $1.9 \pm 0.9$ & $0.009 \pm 0.005$ & $0.0013 \pm 0.0007$ \\
\hline S4TMJ $1403+3309$ & $14: 03: 09.67+33: 09: 17.80$ & 0.06 & 0.77 & 9 & $68 \%$ & $\ldots$ & $\ldots$ & $\ldots$ & $\ldots$ \\
\hline S4TMJ $1430+6104$ & $14: 30: 34.77+61: 04: 04.80$ & 0.17 & 0.65 & 11 & $53 \%$ & $\ldots$ & $\ldots$ & $\ldots$ & $\ldots$ \\
\hline S4TMJ $1433+2835$ & $14: 33: 51.63+28: 35: 16.40$ & 0.09 & 0.41 & 10 & $37 \%$ & $5.3 \pm 1.3$ & $2.2 \pm 1.1$ & $0.011 \pm 0.006$ & $0.0015 \pm 0.0008$ \\
\hline S4TMJ $1541+3642$ & $15: 41: 22.27+36: 42: 31.70$ & 0.14 & 0.74 & 16 & $53 \%$ & $5.9 \pm 0.9$ & $6.9 \pm 3.2$ & $0.028 \pm 0$ & $0.0035 \pm 0.0016$ \\
\hline S4TMJ $1543+2202^{*}$ & $39.94+22: 02: 23.30$ & 0.27 & 0.40 & 3 & $70 \%$ & $10.5 \pm 1.3$ & $6.7 \pm 3.1$ & $0.033 \pm 0$ & $0.0047 \pm 0$ \\
\hline S4TMJ $1550+2020^{*}$ & $15: 50: 10.62+20: 20: 13.50$ & 0.14 & 0.35 & 2 & $35 \%$ & $6.3 \pm 1.2$ & $7.2 \pm 3.6$ & $0.038 \pm 0.018$ & $0.0053 \pm 0.0026$ \\
\hline S4TMJ $1553+3004$ & $15: 53: 16.14+30: 04: 25.70$ & 0.16 & 0.57 & 5 & $75 \%$ & $4.4 \pm 0.9$ & $5.5 \pm 2.7$ & $0.024 \pm 0.012$ & $0.0032 \pm 0.0016$ \\
\hline S4TMJ $1607+2147$ & $16: 07: 40.50+21: 47: 11.00$ & 0.21 & 0.49 & 2 & $53 \%$ & $1.6 \pm 0.6$ & $4.6 \pm 2.6$ & $0.022 \pm 0.012$ & $0.0029 \pm 0.0017$ \\
\hline S4TMJ $1633+1441$ & $16: 33: 44.16+14: 41: 54.90$ & 0.13 & 0.58 & 19 & $51 \%$ & $5.5 \pm 1.1$ & $2.6 \pm 1.3$ & $0.011 \pm 0.006$ & $0.0015 \pm 0.0007$ \\
\hline S4TMJ 2309-0039 & $23: 09: 46.36-00: 39: 12.90$ & 0.29 & 1.00 & 4 & $37 \%$ & $2.1 \pm 1.1$ & $46.1 \pm 32.3$ & $0.161 \pm 0.113$ & $0.0190 \pm 0.0133$ \\
\hline S4TMJ $2324+0105$ & $23: 24: 27.77+01: 05: 58.50$ & 0.19 & 0.28 & 8 & $73 \%$ & $\ldots$ & $\ldots$ & $\ldots$ & $\ldots$ \\
\hline BELLSJ $0151+0049$ & 01:51:07.37+00:49:09.00 & 0.52 & 1.36 & 7 & $43 \%$ & $\ldots$ & $\ldots$ & $\ldots$ & $\ldots$ \\
\hline BELLSJ $0747+4448$ & $07: 47: 34.75+44: 48: 59.30$ & 0.44 & 0.90 & 26 & $51 \%$ & $0.8 \pm 0.2$ & $1.2 \pm 0.6$ & $0.004 \pm 0.002$ & $0.0005 \pm 0.0003$ \\
\hline BELLSJ $0747+5055$ & $07: 47: 24.12+50: 55: 37.50$ & 0.44 & 0.90 & 3 & $31 \%$ & $3.9 \pm 1.7$ & $81.6 \pm 50.7$ & $0.301 \pm 0.187$ & $0.0365 \pm 0.0226$ \\
\hline BELLSJ $0801+4727$ & $08: 01: 05.30+47: 27: 49.61$ & 0.48 & 1.52 & 4 & $50 \%$ & $3.5 \pm 0.7$ & $229.8 \pm 113.0$ & $0.639 \pm 0$ & $0.0682 \pm 0.0335$ \\
\hline BELLSJ $0830+5116$ & $08: 30: 49.73+51: 16: 31.80$ & 0.53 & 1.33 & 6 & $23 \%$ & $2.3 \pm 1.1$ & $134.0 \pm 86.5$ & $0.402 \pm 0.260$ & $0.0444 \pm 0.0287$ \\
\hline BELLSJ 0944-0147 & $09: 44: 27.47-01: 47: 42.40$ & 0.54 & 1.18 & 5 & $37 \%$ & $1.3 \pm 1.0$ & $34.5 \pm 30.6$ & $0.111 \pm 0.098$ & $0.0126 \pm 0.0112$ \\
\hline BELLSJ 1159-0007 & $11: 59: 44.63-00: 07: 28.20$ & 0.58 & 1.35 & 8 & $43 \%$ & $3.7 \pm 0.6$ & $81.4 \pm 38.8$ & $0.243 \pm 0.116$ & $0.0268 \pm 0.0128$ \\
\hline BELLSJ $1215+0047$ & $12: 15: 04.44+00: 47: 26.00$ & 0.64 & 1.30 & 3 & $14 \%$ & $1.8 \pm 0.8$ & $266.6 \pm 165.3$ & $0.812 \pm 0.504$ & $0.0903 \pm 0.0560$ \\
\hline BELLSJ $1221+3806$ & $12: 21: 51.92+38: 06: 10.50$ & 0.53 & 1.28 & 4 & $36 \%$ & $1.8 \pm 0.7$ & $97.2 \pm 56.4$ & $0.298 \pm 0.173$ & $0.0332 \pm 0.0193$ \\
\hline BELLSJ 1234-0241 & $12: 34: 27.99-02: 41: 29.60$ & 0.49 & 1.02 & 2 & $44 \%$ & $1.8 \pm 0.3$ & $50.9 \pm 24.3$ & $0.177 \pm 0.084$ & $0.0208 \pm 0.0099$ \\
\hline BELLSJ 1318-0104 & $13: 18: 29.39-01: 04: 21.60$ & 0.66 & 1.40 & 7 & $46 \%$ & $2.2 \pm 0.6$ & $68.3 \pm 36.3$ & $0.199 \pm 0.106$ & $0.0218 \pm 0.0116$ \\
\hline BELLSJ $1337+3620$ & $13: 37: 51.31+36: 20: 18.10$ & 0.56 & 1.18 & 12 & $15 \%$ & $1.6 \pm 0.7$ & $46.4 \pm 29.1$ & $0.149 \pm 0.093$ & $0.0169 \pm 0.0106$ \\
\hline BELLSJ $1349+3612$ & $13: 49: 10.30+36: 12: 39.70$ & 0.44 & 0.89 & 5 & $36 \%$ & $2.1 \pm 0.3$ & $23.6 \pm 11.2$ & $0.087 \pm 0.041$ & $0.0106 \pm 0.0050$ \\
\hline BELLSJ $1352+3216$ & $13: 52: 18.99+32: 16: 51.80$ & 0.46 & 1.03 & 6 & $9 \%$ & $1.0 \pm 0.3$ & $65.5 \pm 33.5$ & $0.225 \pm 0.115$ & $0.0265 \pm 0.0136$ \\
\hline BELLSJ $1522+2910$ & $15: 22: 09.54+29: 10: 21.90$ & 0.56 & 1.31 & 6 & $43 \%$ & $3.8 \pm 0.8$ & $114.8 \pm 57.2$ & $0.348 \pm 0.173$ & $0.0385 \pm 0.0192$ \\
\hline BELLSJ $1541+1812$ & $15: 41: 18.56+18: 12: 35.10$ & 0.56 & 1.11 & 4 & $45 \%$ & $\ldots$ & $\ldots$ & $\ldots$ & $\ldots$ \\
\hline BELLSJ $1542+1629$ & $15: 42: 46.33+16: 29: 51.80$ & 0.35 & 1.02 & 3 & $25 \%$ & $1.5 \pm 1.0$ & $57.2 \pm 46.0$ & $0.198 \pm 0.159$ & $0.0233 \pm 0.0187$ \\
\hline BELLSJ $1545+2748$ & $15: 45: 03.57+27: 48: 05.30$ & 0.52 & 1.29 & 4 & $23 \%$ & $\ldots$ & $\ldots$ & $\ldots$ & $\ldots$ \\
\hline
\end{tabular}


Table 1

(Continued)

\begin{tabular}{|c|c|c|c|c|c|c|c|c|c|}
\hline $\begin{array}{l}\text { Target } \\
\text { (1) }\end{array}$ & $\begin{array}{l}\text { Position } \\
\text { (2) }\end{array}$ & $\begin{array}{l}z_{L} \\
(3)\end{array}$ & $\begin{array}{l}z_{S} \\
(4)\end{array}$ & (5) & $\begin{array}{r}f_{\text {fiber }} \\
(6)\end{array}$ & $\begin{array}{c}F_{[\mathrm{O} \text { II] }} \\
(7)\end{array}$ & $\begin{array}{c}\mathrm{SFR} \\
M_{\odot} \mathrm{yr}^{-1} \\
(8)\end{array}$ & $\begin{array}{c}N_{\mathrm{cc}} \\
\mathrm{yr}^{-1} \\
(9)\end{array}$ & $\begin{array}{c}N_{\text {Ia }} \\
\mathrm{yr}^{-1} \\
(10)\end{array}$ \\
\hline BELLSJ 1601+2138 & $16: 01: 13.27+21: 38: 33.90$ & 0.54 & 1.45 & 3 & $26 \%$ & $1.3 \pm 0.8$ & $151.4 \pm 119.4$ & $0.433 \pm 0.342$ & $0.0468 \pm 0.0369$ \\
\hline BELLSJ 1611+1705 & $16: 11: 09.80+17: 05: 26.60$ & 0.48 & 1.21 & 3 & $34 \%$ & $2.1 \pm 0.3$ & $121.7 \pm 58.1$ & $0.385 \pm 0.184$ & $0.0436 \pm 0.0208$ \\
\hline BELLSJ 1631+1854 & $16: 31: 50.33+18: 54: 04.10$ & 0.41 & 1.09 & 19 & $12 \%$ & $3.6 \pm 0.5$ & $59.5 \pm 27.9$ & $0.200 \pm 0.094$ & $0.0232 \pm 0.0109$ \\
\hline BELLSJ 1637+1439 & $16: 37: 14.58+14: 39: 30.10$ & 0.39 & 0.87 & 11 & $41 \%$ & $0.8 \pm 0.2$ & $3.2 \pm 1.6$ & $0.012 \pm 0.006$ & $0.0015 \pm 0.0007$ \\
\hline BELLSJ 2122+0409 & $21: 22: 52.04+04: 09: 35.50$ & 0.63 & 1.45 & 6 & $19 \%$ & $0.9 \pm 0.5$ & $93.4 \pm 69.2$ & $0.267 \pm 0.197$ & $0.0288 \pm 0.0213$ \\
\hline BELLSJ 2125+0411 & $21: 25: 10.67+04: 11: 31.60$ & 0.36 & 0.98 & 4 & $18 \%$ & $1.3 \pm 0.2$ & $42.0 \pm 20.2$ & $0.149 \pm 0.071$ & $0.0177 \pm 0.0085$ \\
\hline BELLSJ 2303+0037 & $23: 03: 35.17+00: 37: 03.20$ & 0.46 & 0.94 & 7 & $28 \%$ & $0.9 \pm 0.2$ & $9.2 \pm 4.7$ & $0.033 \pm 0.017$ & $0.0040 \pm 0.0020$ \\
\hline
\end{tabular}

Note. Column 1 is the system name with leading letters indicating the specific survey program. Column 2 is the system position in right ascension and declination. Columns 3 and 4 are the lens and source redshifts inferred from the SDSS/BOSS spectrum. Column 5 provides the inferred total magnification from the best-fit lens model. Columns 6 and 7 are the fraction of source flux and apparent [O II] flux (in units of $10^{-16} \mathrm{erg} \mathrm{s}^{-1} \mathrm{~cm}^{2}$ ) collected by the SDSS/BOSS fiber. Columns 8 is the intrinsic SFR estimated from [O II] flux. Columns 9 and 10 are the core-collapse SN rate, and Type-Ia SN rate inferred from the SFR. Asterisked are systems with simultaneous $\mathrm{H} \alpha$ detections.

\subsection{Intrinsic [O II] Flux Determination}

To enable SFR estimation, we first determine the intrinsic integrated [O II] flux of each lensed source, which has been shown to be an empirical SFR indicator (e.g., Gallagher et al. 1989; Kennicutt 1992, 1998; Hogg et al. 1998; Jansen et al. 2001; Cardiel et al. 2003; Hopkins et al. 2003; Kewley et al. 2004; Moustakas et al. 2006; Gilbank et al. 2010; Hayashi et al. 2013). We note that a small fraction $(\sim 10 \%)$ of our lensed sources also shows $\mathrm{H} \alpha$ emission in the optical spectroscopic data, which is, in principle, a better SFR indicator than [O II] (e.g., Kennicutt 1983, 1998; Moustakas et al. 2006). Nevertheless, for uniformity, we only use [O II] flux for the SFR estimation for the entire sample.

The observed integrated [O II] flux, $F_{[\mathrm{O} \mathrm{II}}$, is measured from the SDSS or BOSS spectrum, collected by a fiber centered on the lens galaxy. The lens-galaxy spectrum is first subtracted following procedures in Bolton et al. (2006a, 2012b), Brownstein et al. (2012), and Shu et al. (2016a). The [O II] emission profile is then fitted by a double Gaussian plus a first-order polynomial (i.e., a line). The ratio of the central wavelengths of the two Gaussians is fixed to $3727.09 / 3729.88$. The parameter optimization is done using the Levenberg-Marquardt algorithm implemented in the MPFIT package (Markwardt 2009). The best-fit integrated [O II] fluxes of the lensed sources are provided in Column (7) of Tabel 1. Note that we choose not to report results for 18 systems for which the inferred [O II] flux error is larger than the best-fit [O II] flux. Calculations of the SFR and SN rates in the rest of the paper are based on the remaining 110 strong-lens systems after excluding these 18 .

To obtain the intrinsic [O II] integrated flux, we correct for the lensing magnification and fiber loss, as inferred from the strong-lens models. For the S4TM lensed sources, we use the lens models from Shu et al. (2017). We remodel the SLACS strong-lens systems following the same methodology as Shu et al. (2017) because their lensing magnifications are not publicly available. For consistency, we also remodel the BELLS strong-lens systems in the same way. We calculate the total magnification, $\mu$, for each lens system. To quantify the uncertainty in $\mu$ due to different lens-modeling tools, we compare our values to the published values in Brownstein et al. (2012) for the 25 BELLS strong-lens systems. The average $\mu$ ratio, defined as $\mu_{\text {this work }} / \mu_{\text {Brownstein2012 }}$, is 0.94 , indicating relatively little bias in the $\mu$ determination. The standard deviation of the $\mu$-ratio distribution is 0.16 , which we adopt as the fractional uncertainty in $\mu$. The fraction of the lensed source flux captured by either the $3^{\prime \prime}$-diameter fiber (for SLACS and S4TM lenses) or the $2^{\prime \prime}$-diameter fiber (for BELLS lenses), denoted as $f_{\text {fiber }}$, is determined by convolving the best-fit model of the lensed images obtained from the HST imaging data with the ground-based point-spread function of the spectroscopic observation. A top-hat kernel matched with the specific fiber size is then applied to the convolved image to determine $f_{\text {fiber }}$. Columns (5) and (6) of Tabel 1 list $\mu$ and $f_{\text {fiber }}$ values. The intrinsic source [O II] integrated flux is then computed as

$$
F_{[\mathrm{O} \text { II }]}^{\text {intric }}=F_{[\mathrm{O} \mathrm{II}]} / f_{\text {fiber }} / \mu \text {. }
$$

Note that all the strong-lens models, and hence the derived $\mu$ and $f_{\text {fiber }}$ quantities, are based on HST F814W wide-band imaging data, which covers roughly $7500 \AA-9500 \AA$ in the observed frame. In the source rest frame, these HST observations probe continuum emission at $4500 \AA-6000 \AA$ (for the SLACS and S4TM lensed sources with an average redshift of 0.6) and $3400 \AA-4300 \AA$ (for the BELLS lensed sources with an average redshift of 1.2). Strictly speaking, the derived $\mu$ and $f_{\text {fiber }}$ apply to the source continuum-emitting regions. We nevertheless adopt these as the values for the source [O II] emitting regions simply because no other data are available for better determinations of $\mu$ and $f_{\text {fiber }}$ of [O II] emission. At least for BELLS lensed sources, this approximation should be reasonably accurate because the [O II] emission is driven by the ultraviolet (UV) radiation from massive young stars, which are also primarily responsible for the UV continuum emission around $3400 \AA-4300 \AA$.

The distributions of $\mu$ and $f_{\text {fiber }}$ for the full sample, as well as for each individual lens sample, are shown in Figure 1. The $\mu$ histogram of the full sample suggests that the probability density function (PDF) of $\log _{10} \mu$ can be well characterized by a skew-normal function as

$$
p\left(\log _{10} \mu\right)=\frac{2}{\sigma_{\log _{10} \mu}} \phi\left(\frac{\log _{10}\left(\mu / \mu_{0}\right)}{\sigma_{\log _{10} \mu}}\right) \Phi\left(\alpha\left(\frac{\log _{10}\left(\mu / \mu_{0}\right)}{\sigma_{\log _{10} \mu}}\right)\right),
$$

in which $\phi(x)$ and $\Phi(x)$ are the PDF and cumulative density function of a Gaussian distribution, respectively. The three parameters $\mu_{0}, \sigma_{\log _{10} \mu}$, and $\alpha$ describe the location, scale, and 

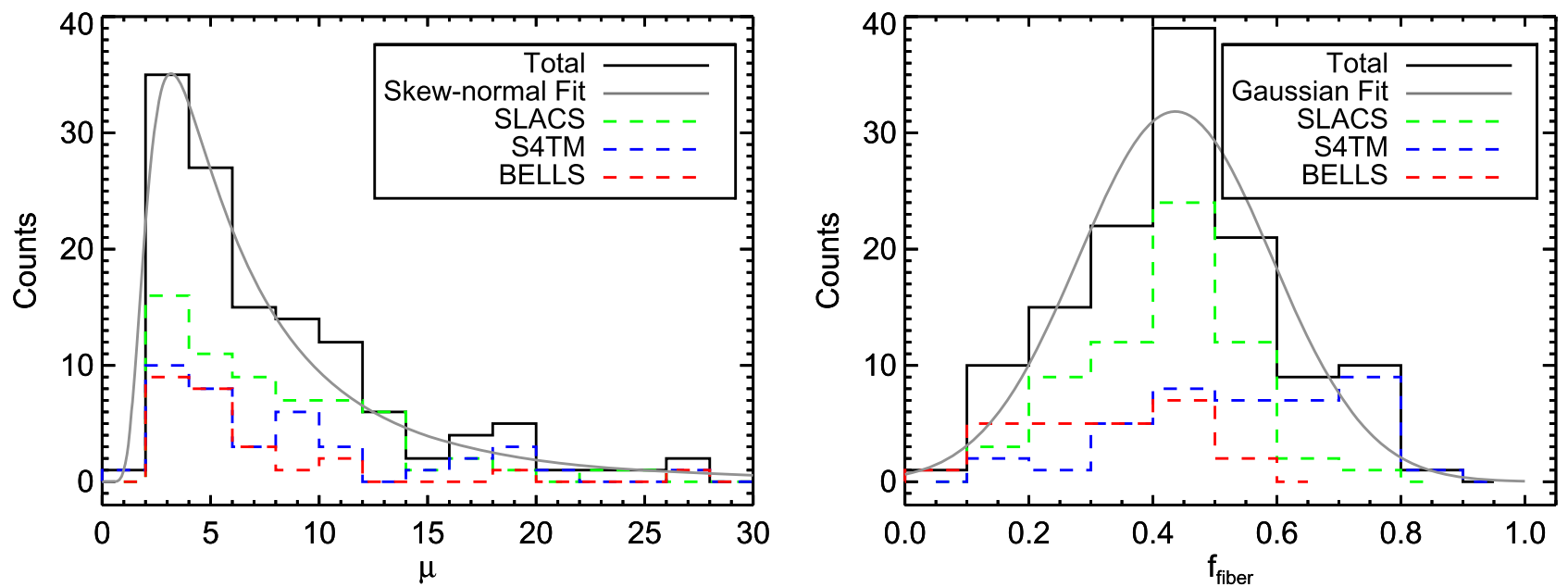

Figure 1. Histograms of the total magnification, $\mu$, and the fraction of flux captured by the fiber, $f_{\text {fiber }}$, derived from the best-fit lens models for the full lens compilation (solid black) as well as the three individual lens samples (dashed colors). The gray lines represent the best analytical fits to the full-sample histograms.

degree of skewness of the $\log _{10} \mu$ PDF (respectively). The gray curve in the left panel of Figure 1 shows the best skew-normal fit to the histogram of the full sample (in black) with

$$
\begin{gathered}
\mu_{0}=5.0 \pm 1.9, \\
\sigma_{\log _{10} \mu}=0.91 \pm 0.09, \\
\alpha=2.5 \pm 1.0 .
\end{gathered}
$$

We find that the $\mu$-distributions of the three individual lens samples (as shown by the dashed histograms in color) show no significant deviation from that of the full sample.

The $f_{\text {fiber }}$ PDF of the full sample can be approximated as a Gaussian function with best-fit mean $f_{\text {fiber,0}}$ and standard deviation $\sigma_{f_{\text {fiber }}}$ parameters given by

$$
\begin{gathered}
f_{\text {fiber }, 0}=0.44 \pm 0.02, \\
\sigma_{\text {fiber }}=0.16 \pm 0.02 .
\end{gathered}
$$

The distributions of the individual lens samples are similar at small $f_{\text {fiber }}$ values, but become significantly different at large $f_{\text {fiber. }}$ The BELLS lens sample does not have any system with $f_{\text {fiber }}>60 \%$. This is primarily related to the fact that the fiber size for BELLS lens systems is more than $30 \%$ smaller than that of SLACS and S4TM lens systems. On the other hand, over $40 \%$ of the S4TM lens systems have $f_{\text {fiber }}>60 \%$, which is much larger than the fraction for the SLACS lens systems $(<5 \%)$ with the same fiber size. We attribute this difference to the fact that the S4TM lens galaxies are, on average, a factor of two less massive than the SLACS lens galaxies as found in Shu et al. (2017). With similar lens and source redshifts, the less massive S4TM lens galaxies form lensed images with smaller angular separations, which are more likely to be covered by the fiber.

\subsection{Star Formation Rate from [O II] Luminosity}

Additional calibration is generally needed for an [O II]derived SFR because [O II] emission is not directly coupled to the ionizing flux alone, but rather is also sensitive to the oxygen abundance. In addition, the intrinsic [O II] luminosity cannot be accurately inferred without correction for dust extinction. Various studies have attempted to calibrate the [O II]-derived
SFR against other observables such as oxygen abundance, $[\mathrm{O} \mathrm{II}] / \mathrm{H} \alpha$ line ratio, broadband luminosity, stellar mass, and [O II] equivalent width (e.g., Hicks et al. 2002; Kewley et al. 2004; Moustakas et al. 2006; Argence \& Lamareille 2009; Gilbank et al. 2010; Mostek et al. 2012; Hayashi et al. 2013; Talia et al. 2015). However, due to the limited data available for the lensed sources, we cannot perform a full calibration on a system-by-system basis. Instead, we analyze the subsample of our lens compilation with detected $\mathrm{H} \alpha$ emission, and apply the resulting calibration to the full sample.

$\mathrm{H} \alpha$ luminosity is one of the most reliable SFR indicators, since it scales directly with ionizing flux. A commonly used conversion due to Kennicutt (1998) is

$$
\frac{\mathrm{SFR}}{M_{\odot} \mathrm{yr}^{-1}}=7.9 \times 10^{-42} \frac{L_{\mathrm{H} \alpha}}{\mathrm{erg} \mathrm{s}^{-1}},
$$

which assumes no dust and a Salpeter initial mass function (IMF, Salpeter 1955). Here, $L_{\mathrm{H} \alpha}$ is the intrinsic $\mathrm{H} \alpha$ luminosity. In the presence of dust, we can rewrite this relation as

$$
\frac{\mathrm{SFR}}{M_{\odot} \mathrm{yr}^{-1}}=7.9 \times 10^{-42} \frac{10^{0.4 A_{\mathrm{H} \alpha}} L_{\mathrm{H} \alpha}^{\mathrm{obs}}}{\mathrm{erg} \mathrm{s}^{-1}},
$$

in which $L_{\mathrm{H} \alpha}^{\mathrm{obs}}$ is the observed $\mathrm{H} \alpha$ luminosity and $A_{\mathrm{H} \alpha}$ is the dust extinction at the wavelength of $\mathrm{H} \alpha$. We can further transform the conversion as

$$
\frac{\mathrm{SFR}}{M_{\odot} \mathrm{yr}^{-1}}=\frac{10^{0.4 A_{\mathrm{H} \alpha}}}{F_{[\mathrm{O} \mathrm{III}} / F_{\mathrm{H} \alpha}} \frac{L_{[\mathrm{OII}]}^{\mathrm{obs}}}{1.27 \times 10^{41} \mathrm{erg} \mathrm{s}^{-1}},
$$

in which $F_{[\mathrm{O} \mathrm{II}]}, F_{\mathrm{H} \alpha}$, and $L_{[\mathrm{O} \text { II] }}^{\mathrm{ob}}$ are the observed, dustextinction uncorrected [O II] flux, $\mathrm{H} \alpha$ flux, and [O II] luminosity, respectively. The [O II] luminosity is simply computed from the [O II] flux $F_{[\mathrm{O} \text { II }]}^{\text {intric }}$ as

$$
L_{[\mathrm{O} \mathrm{II}]}=4 \pi D_{L}^{2}\left(z_{S}\right) F_{[\mathrm{O} \mathrm{II}]}^{\mathrm{intrinsic}},
$$

where $D_{L}\left(z_{S}\right)$ is the luminosity distance at the source redshift $z_{S}$.

We use the 12 strong-lens systems with simultaneously detected $\mathrm{H} \alpha$ emissions to establish a fiducial $[\mathrm{O} \mathrm{II}] / \mathrm{H} \alpha$ ratio for the full lens compilation. We fit a single Gaussian plus a first-order polynomial to the observed $\mathrm{H} \alpha$ emission in the 


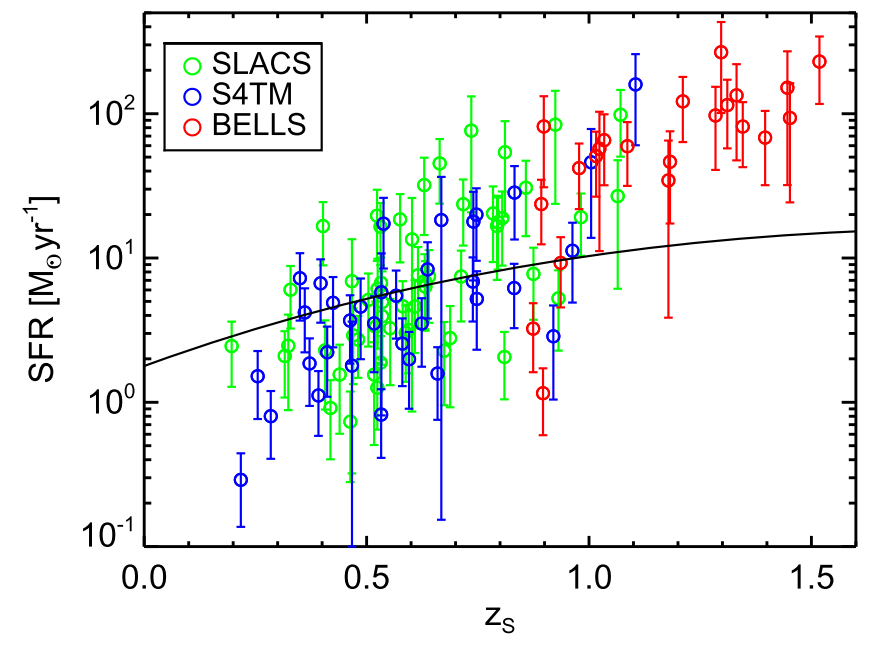

Figure 2. SFR-redshift relation for the SLACS (in green), S4TM (in blue), and BELLS (in red) lensed sources. The black line represents the SFR-redshift relation defined in Equation (16) normalized at $z_{S}=0.9$ where the three lens samples have a substantial overlap.

residual spectrum to obtain the observed $\mathrm{H} \alpha$ flux for these 12 lensed sources. We find that the $\mathrm{H} \alpha /[\mathrm{O} \mathrm{II}]$ flux ratio is centered at 1.2 with a scatter of 0.5 , consistent with the results in Hayashi et al. (2013), who studied a sample of $z=1.47$ [O II] emitters. Furthermore, the typical [O II] luminosity level of the full lensed source sample is about $(4-60) \times 10^{41} \mathrm{erg} \mathrm{s}^{-1}$, which is also similar to that of the [O II] emitter sample in Hayashi et al. (2013). Based on the $\mathrm{H} \alpha /[\mathrm{O}$ II $]$ flux ratio, Hayashi et al. (2013) estimated the dust extinction at $\mathrm{H} \alpha$ to be 0.35 mag using the empirical relation from Sobral et al. (2012). We thus assume that the $F_{[\mathrm{OI}]} / F_{\mathrm{H} \alpha}$ ratio for the full lens compilation is $0.83 \pm 0.35$, and $A_{\mathrm{H} \alpha}$ is $0.35 \mathrm{mag}$. The conversion from $L_{[\mathrm{O} \text { II] }}$ to SFR now becomes

$$
\frac{\mathrm{SFR}}{M_{\odot} \mathrm{yr}^{-1}}=(1.31 \pm 0.55) \times 10^{-41} L_{[\mathrm{O} \text { II }]} .
$$

Note that this conversion is obtained assuming a Salpeter IMF, while recent studies have suggested that the stellar IMF may vary with galaxy properties (e.g., Auger et al. 2010; Treu et al. 2010; van Dokkum \& Conroy 2010; Strader et al. 2011; Cappellari et al. 2012; Sonnenfeld et al. 2012; Spiniello et al. 2012; Conroy et al. 2013; Ferreras et al. 2013; la Barbera et al. 2013; Tortora et al. 2013; Brewer et al. 2014; Spiniello et al. 2014; Shu et al. 2015; Li et al. 2017). An extra factor of 0.67 would need to be included to convert the reported SFRs to values under the assumption of a Kroupa IMF (Kroupa 2001), to account for the mass ratio between the two IMFs for the same amount of ionizing radiation (e.g., Brinchmann et al. 2004; Gilbank et al. 2010).

The estimated intrinsic SFRs for the lensed sources from the above analysis are provided in Column (8) of Table 1. The uncertainty in the SFR takes into account uncertainties in the apparent [O II] flux, total magnification, and conversion factor from [O II] luminosity to SFR. Figure 2 shows the estimated SFR against redshift of each individual lensed sources from the three lens samples in different colors. We find that the SFRs for the lensed sources range from $0.3 M_{\odot} \mathrm{yr}^{-1}$ to $267 M_{\odot} \mathrm{yr}^{-1}$. The median SFRs for the SLACS, S4TM, and BELLS lensed sources are $6 M_{\odot} \mathrm{yr}^{-1}, \quad 5 M_{\odot} \mathrm{yr}^{-1}$, and $68 M_{\odot} \mathrm{yr}^{-1}$, respectively. Furthermore, there is a strong correlation between
SFR and source redshift, which is primarily a selection bias, since all the lens systems are selected to have their apparent [O II] fluxes above a certain signal-to-noise ratio $(\mathrm{S} / \mathrm{N})$ threshold. One can see from Column (7) in Table 1 that the apparent [O II] flux level is indeed similar across the three lens samples. As a result, the intrinsic [O II] luminosities, and hence the derived SFRs, are higher at progressively higher redshifts. To further contextualize this, we overplot the cosmic star formation history trend given by Madau \& Dickinson (2014, i.e., Equation (16) in this work) in black in Figure 2. This trend is normalized such that the SFR at $z_{S}=0.9$, where the three lens samples overlap, is equal to the median SFR of the lensed sources within $0.85<z_{S}<0.95$. It can be seen that the lensed galaxies in our combined sample are more intensely starforming at higher redshifts, even after taking into account the cosmic evolution of the SFR.

\subsection{Supernova Rates from Star Formation Rate}

\subsubsection{Core-collapse Supernova Rate}

The rate of CCSNe is proportional to recent SFRs (e.g., Dahlén \& Fransson 1999; Oguri \& Marshall 2010) according to the relation

$$
N_{\mathrm{cc}}=\frac{1}{1+z_{S}} k_{\mathrm{cc}} \times \mathrm{SFR},
$$

in which $k_{\mathrm{cc}}$ is the number of CCSNe per unit mass, and $N_{\mathrm{cc}}$ is the occurrence rate of CCSNe per year. An extra factor of $\left(1+z_{S}\right)^{-1}$ is applied to account for the time dilation because the derived SFRs are values in the lensed source rest frame while we are interested in the $\mathrm{SN}$ rates in the observed frame (the same factor is applied to the SNIa rate calculation below as well). Effectively, $k_{\mathrm{cc}}$ is the number of CCSN progenitors per unit mass, averaged across all recently formed stars. Assuming a particular IMF, $\phi(m), k_{\mathrm{cc}}$ can be computed as

$$
k_{\mathrm{cc}}=\frac{\int_{M_{1}}^{M_{2}} \phi(M) d M}{\int_{M_{\min }}^{M_{\max }} M \phi(M) d M},
$$

in which $M_{\min }$ and $M_{\max }$ are the lower and upper cutoff masses for the considered IMF, and $M_{1}-M_{2}$ represents the mass range of CCSN progenitors. To be consistent with the IMF choice for the SFR estimation, we choose a Salpeter IMF with $M_{\min }=0.1 M_{\odot}$ and $M_{\max }=125 M_{\odot}$. The mass range of CCSN progenitors is assumed to be $8-50 M_{\odot}$, since more massive stars likely form black holes instead of SNe (e.g., Tsujimoto et al. 1997; Hopkins \& Beacom 2006; Oguri \& Marshall 2010; Dahlen et al. 2012; Melinder et al. 2012). Under these assumptions, $k_{\mathrm{cc}}$ is computed to be $0.0070 M_{\odot}^{-1}$. Note that Strolger et al. (2015) empirically found $k_{\mathrm{cc}}$ to be $0.0091 \pm 0.0017 M_{\odot}^{-1}$, broadly consistent with the value we adopt here.

\subsubsection{Type Ia Supernova Rate}

In principle, the occurrence rate of SNIa can be similarly estimated (e.g., Dahlén \& Fransson 1999; Oguri \& 
Marshall 2010) according to

$$
\begin{gathered}
N_{\mathrm{Ia}}=\eta C_{\mathrm{Ia}} \frac{\int_{t_{\min }}^{t\left(z_{S}\right)} \operatorname{SFR}\left(t\left(z_{S}\right)-t_{\mathrm{D}}\right) f_{\mathrm{D}}\left(t_{\mathrm{D}} ; \tau\right) d t_{\mathrm{D}}}{\left(1+z_{S}\right) \int_{t_{\min }}^{t(0)} f_{\mathrm{D}}\left(t_{\mathrm{D}} ; \tau\right) d t_{\mathrm{D}}} \\
C_{\mathrm{Ia}}=\frac{\int_{M_{1}^{\prime}}^{M_{2}^{\prime}} \phi(M) d M}{\int_{M_{\text {min }}}^{M_{\max }} m \phi(M) d M},
\end{gathered}
$$

in which $M_{1}^{\prime}=3 M_{\odot}$ and $M_{2}^{\prime}=8 M_{\odot}$ are the mass limits of SNIa progenitors, $\eta$ is the fraction of progenitors that successfully explode as an SNIa, and $f_{\mathrm{D}}\left(t_{\mathrm{D}} ; \tau\right)$ is the delaytime $\left(t_{\mathrm{D}}\right)$ distribution with a characteristic timescale $\tau$ and a minimum delay time $t_{\text {min }}$. However, this conversion is subject to significant systematics. In particular, $\eta, \tau$, and $f_{\mathrm{D}}\left(t_{\mathrm{D}} ; \tau\right)$ are not well constrained. Usually, $\eta$ is calibrated by matching the predicted rate at $z=0$ to the observed SNIa rate in the local universe. The typical value for $\eta$ is $4 \%-10 \%$ (e.g., Madau et al. 1998; Dahlen et al. 2004; Hopkins \& Beacom 2006; Maoz et al. 2012), which depends on the assumed delay-time distribution and $\tau$ value. To be conservative, we choose $\eta=4 \%$. Again choosing a Salpeter IMF, we have

$$
N_{\mathrm{Ia}}=0.00084 M_{\odot}^{-1} \frac{\int_{t_{\min }}^{t\left(z_{S}\right)} \operatorname{SFR}\left(t\left(z_{S}\right)-t_{\mathrm{D}}\right) f_{\mathrm{D}}\left(t_{\mathrm{D}} ; \tau\right) d t_{\mathrm{D}}}{\left(1+z_{S}\right) \int_{t_{\min }}^{t(0)} f_{\mathrm{D}}\left(t_{\mathrm{D}} ; \tau\right) d t_{\mathrm{D}}} .
$$

We assume the SFHs of lensed sources, $\operatorname{SFR}(t(z))$, follow the best-fit functional form of the observed cosmic SFH in Madau \& Dickinson (2014), and use the measured SFRs at $z_{S}$ as normalizations

$$
\operatorname{SFR}(t(z))=\operatorname{SFR}\left(z_{S}\right)\left(\frac{1+z(t)}{1+z_{S}}\right)^{2.7} \frac{\left[1+\left(\frac{1+z_{S}}{2.9}\right)^{5.6}\right]}{\left[1+\left(\frac{1+z(t)}{2.9}\right)^{5.6}\right]}
$$

As will be shown later, the SNIa rates in this sample are primarily determined by the most recent SFRs, and therefore different assumptions on the SFHs will only introduce minor variations in the estimated SNIa rates that are negligible compared to the overall uncertainties. The delay-time distribution is assumed to be a simple power law with no minimum delay time, as suggested in Maoz et al. (2012):

$$
f_{\mathrm{D}}\left(t_{\mathrm{D}} ; \tau\right) \propto t_{\mathrm{D}}^{-1.07}
$$

The rates of CCSN and SNIa can now be estimated following Equations (11) and (15), and are given in Columns (9) and (10) of Table 1 for each individual lensed source. The rate of SNIa, $N_{\text {Ia }}$, is 0.0002-0.09 per year in the individual lensed galaxies, and the median $N_{\text {Ia }}$ for the SLACS, S4TM, and BELLS lensed source are 0.004, 0.003, and 0.03 per year, respectively. The rate of CCSN in individual lens galaxies, $N_{\mathrm{cc}}$, is $0.002-0.8$ per year, and the median $N_{\mathrm{cc}}$ for the SLACS, S4TM, and BELLS lensed sources are $0.03,0.02$, and 0.2 per year. The integrated rate of SNIa across the entire lens compilation is a factor of $\sim 8$ smaller than that of CCSN. Figure 3 shows the predicted rates of both SN types in the lensed source galaxies, as a function of the source redshifts. Because the rates are either directly proportional to (for CCSN) or determined to the leading order (for SNIa) by the SFR, $N_{\text {Ia }}$ and $N_{\mathrm{cc}}$ increase with source redshift as a result of the same selection effect discussed above. This is further evident in the bimodal distributions of $N_{\text {Ia }}$ and $N_{\text {cc }}$ seen in Figure 3, with the two peaks corresponding to the lower-redshift SLACS+S4TM systems and the higher-redshift BELLS systems. For the full lens compilation with 110 strong-lens systems, we expect in total $10.4 \pm 1.1$ strongly lensed CCSN events per year, and $1.23 \pm 0.12$ strongly lensed SNIa events per year.

\section{Rates of Detectable Strongly Lensed Supernovae}

We now proceed to estimate the occurrence rates of strongly lensed $\mathrm{SNe}$ in each lensed source of our lens compilation that could be detected given specific depths. We assume all the detectable $\mathrm{SNe}$ in the lensed galaxies are strongly lensed too. We use depths of two dedicated time-domain facilities-the Zwicky Transient Facility (ZTF) and LSST-as reference survey depths. The single-visit depth is $20.4 \mathrm{mag}$ ( $5 \sigma$ for point source, $R$ band) for ZTF (Bellm 2014), and 24.7 mag (5 $\sigma$ for point source, $r$ band) for LSST (Ivezic et al. 2008). We thus consider "Program1" and "Program2" as two nominal monitoring programs of this lens compilation with cadences of 5 days and single-visit depths of 20.4 mag and 24.7 mag (both $5 \sigma$ for point source) in the SDSS $r$ band (Doi et al. 2010), respectively.

The detectable SN rates depend on the SN luminosity function, dust extinction, survey depth, and lensing magnification. We assume that peak absolute magnitudes in Johnson $B$-band, $M_{B}$, of different SN types are Gaussian distributed with means and standard deviations given by Richardson et al. (2014, Table 2). The relative abundances of CCSN subtypes are taken from the same paper. For each lensed source, we convert the $M_{B}$ distributions of different $\mathrm{SN}$ types to distributions of apparent magnitude in the $r$ band. The conversion between $M_{B}$ and $r$-band apparent magnitude is done based on $\mathrm{SN}$ template spectra with SNCosmo (Barbary 2014), a Python package that takes K correction and distance modulus into account. Specifically, the mean spectrum from Hsiao et al. (2007) is used for SNIa, the spectrum of SN2006ep (Joubert et al. 2006) is used for Ib, the spectrum of SN2004fe (Pugh et al. 2004) is used for Ic, the spectrum of SN2007pg (Sako et al. 2018) is used for IIP and IIL, and the spectrum of SN2006ix (Bassett et al. 2006) is used for IIn. Due to the lack of observed spectra with sufficient UV coverage for IIb, we use the Ib spectrum as an approximation. As justified in the SFR estimations, we assume for each lensed source a constant extinction of $A_{\mathrm{H} \alpha}=0.35 \mathrm{mag}$ and convert $A_{\mathrm{H} \alpha}$ to the extinction value in the observed $r$ band following a Calzetti extinction law (Calzetti et al. 2000). For the redshift range considered in this paper (i.e., 0.2-1.5), the applied extinctions in the observed $r$ band are in the range 0.5-0.8 mag.

The lensing magnification essentially extends the instrumental detection limit by a factor of $2.5 \log _{10} \mu$. Although the total magnifications for the extended source continuum regions have been calculated from the HST data, the expected magnifications of lensed SN images are very uncertain because an $\mathrm{SN}$ is essentially a point source, the magnification of which is extremely sensitive to its actual position in the source plane. It is also possible that the $\mathrm{SN}$ explosion happens away from the bright continuum region of its host galaxy, especially for SNIa. For the sake of simplicity, we adopt for all the sources a fiducial magnification factor of 5 for the most magnified SN 

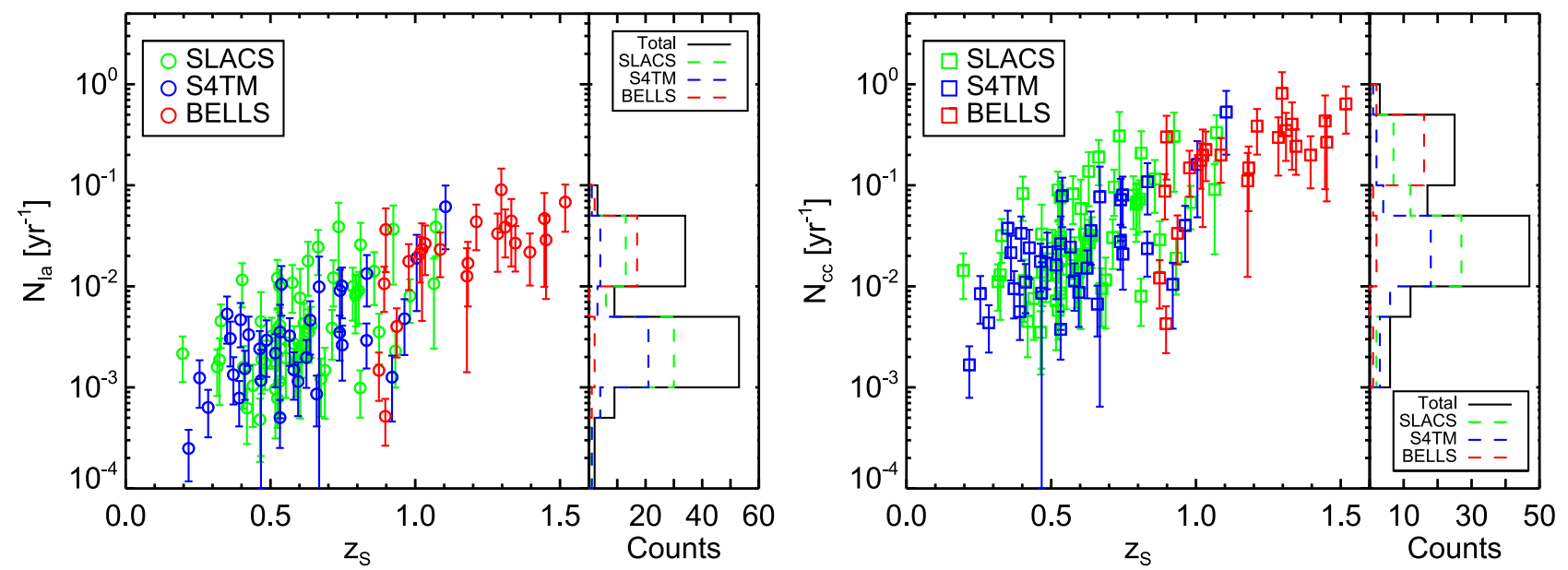

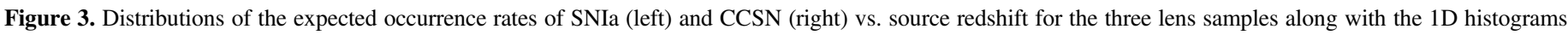
shown on the side.

image, which will extend the detection limit by roughly 1.75 magnitude. We note that this is a conservative assumption as the total magnifications of the three discovered strongly lensed $\mathrm{SNe}$ (all with four lensed images) are 30-100 (Quimby et al. 2014; Kelly et al. 2015; Goobar et al. 2017). We choose not to consider magnitude fluctuations induced to individual lens systems by microlensing (e.g., Dobler \& Keeton 2006) as it would be beyond the scope of this paper.

In this work, we define a lensed SN as detected if its extinction-corrected peak $r$-band apparent magnitude is at least 0.7 mag brighter than the program's effective depth, defined as the single-visit depth plus $2.5 \log _{10} \mu$. The detected fractions of all $\mathrm{SNe}$ are derived from the $r$-band apparent-magnitude distributions for every single-visit depth. The detectable SN rates in each lensed source are determined by multiplying the total SN rates $\left(N_{\text {Ia }}\right.$ and $\left.N_{\text {cc }}\right)$ by these detected fractions. The requirement to be 0.7 mag brighter than the effective survey depth, used in Oguri \& Marshall (2010), allows detections of $\mathrm{SN} \sim 10$ rest-frame days before peak (e.g., Firth et al. 2015; Hoeflich et al. 2017; He et al. 2018; Taddia et al. 2018). Given a cadence of 5 days, which corresponds to $\sim 3$ rest-frame days in our sources, this requirement ensures a reasonable sampling of the SN light curve, especially the early, premaximum color curve that is suggested to be insensitive to the microlensing effect and therefore crucial for accurate time-delay measurements (Goldstein et al. 2018).

Figure 4 shows the expected occurrence rates of detectable strongly lensed SNIa (black solid line) and CCSN (red dashed line) events as functions of the $r$-band equivalent depth. Assuming a fiducial magnification factor of 5 , it suggests that in this lens compilation, Program 1 could detect approximately 0.003 strongly lensed SNIa event and 0.019 strongly lensed CCSN event per year while Program2 could detect 0.49 strongly lensed SNIa event and 2.1 strongly lensed CCSN events per year. In terms of the yields in individual lens samples, the expected occurrence rates of detectable strongly lensed SNIa and CCSN in Program2 are 0.32 and 1.2 event per year for the SLACS sample, 0.12 and 0.5 event per year for the S4TM sample, and 0.05 and 0.4 event per year for the BELLS sample. We also plot the expectations with a fiducial magnification factor of 10 , which are shown by the vertical, gray dotted lines. The numbers increase to 0.016 SNIa and

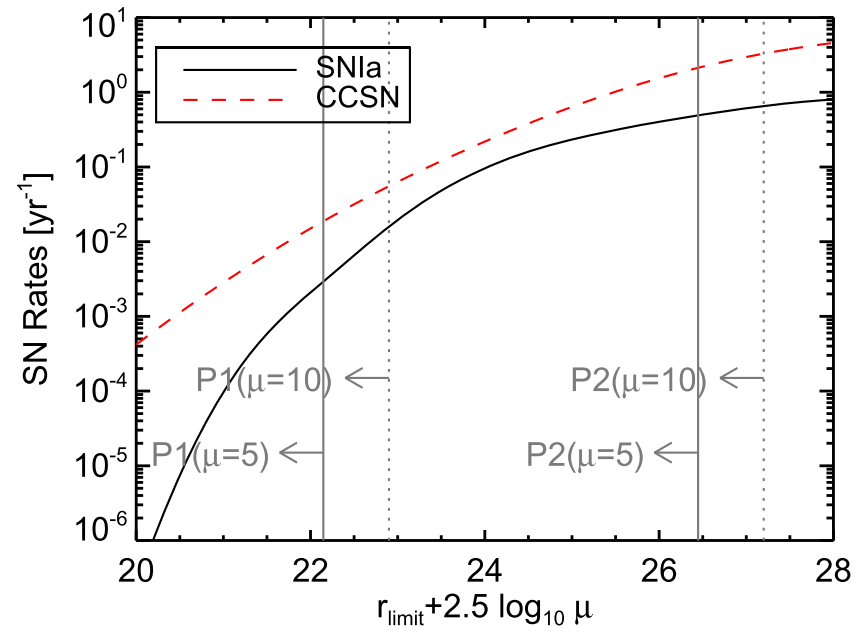

Figure 4. Expected occurrence rates of detectable strongly lensed SNIa (black solid line) and CCSN (red dashed line) events as functions of the $r$-band equivalent depth. The vertical gray lines show the equivalent depths of Program1 and Program2 with fiducial magnification factors of 5 (solid line) and 10 (dotted line)

0.055 CCSN event per year in Program1, and 0.65 SNIa and 3.3 CCSN events per year in Program2.

We further examine the expected occurrence rates of detectable strongly lensed SNIa and CCSN in each individual lensed source in Figure 5. The black symbols show the rates of detectable SNIa (circle) and CCSN (square) as functions of the SN (i.e., source) redshift for Program1 assuming a fiducial magnification factor of 5 , while the red symbols are the same distributions but for Program2. For Program1, the detectabilities of both SN types drop almost monotonically against the SN redshift, even though the overall SN rates are higher at higher redshifts. The reason for this is that the decrease in the fractions of detectable $\mathrm{SNe}$ overwhelms the increase in the overall SN rates at progressively higher redshifts given Program1's shallow detection depth. For Program2 with a deeper detection depth, the detectabilities mildly increase with redshift until about $z=1$ beyond which the detectability of SNIa starts dropping.

Our results suggest that it is better to preferentially focus on lower source-redshift strong-lens systems in a survey with a limiting magnitude similar to that of Program1. A survey with a 


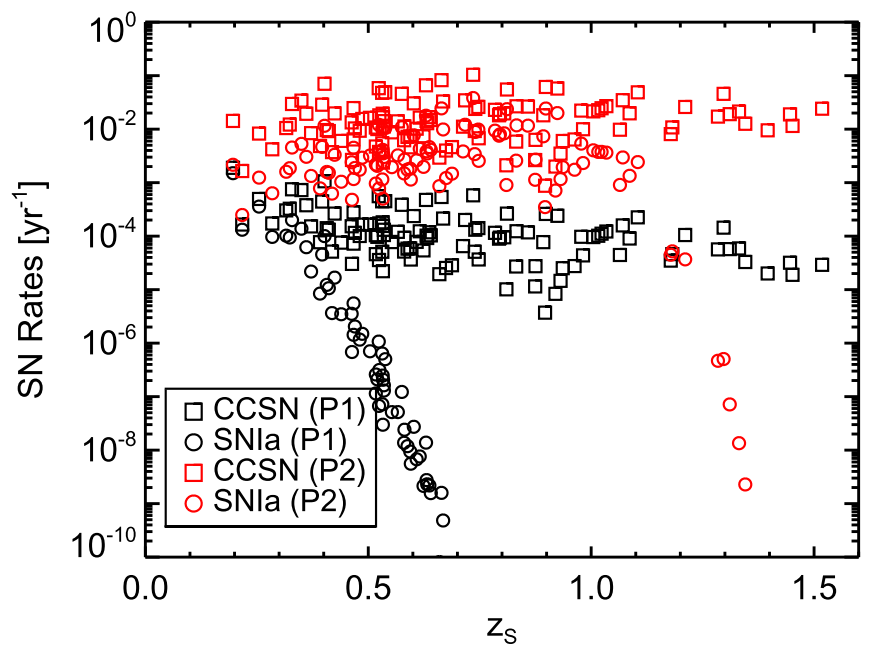

Figure 5. Expected occurrence rates of strongly lensed SNIa (circles) and CCSN (squares) events in individual lensed sources that can be detected in Program1 (black symbols) or Program2 (red symbols) as functions of the source redshift assuming a fiducial magnification factor of 5 .

depth comparable to Program2 is generally sensitive to the entire source-redshift range considered here. To further quantify this, we check the slopes of the detectable SN rateSN redshift relations of both types at different detection depths. We find that the slopes become positive when the detection depth reaches about 24.5 mag or brighter. This implies that the actual strategy of monitoring for strongly lensed $\mathrm{SNe}$ in our lens compilation should be adjusted according to the survey depth.

\section{Discussion}

The biggest systematic uncertainty in our SN-rate estimation is in the conversion from [O II] flux to SFR. Due to the lack of data for full calibrations on a system-by-system basis, we decided to use a subsample with simultaneous detections of $\mathrm{H} \alpha$ emissions from the lensed sources and apply the resulting calibrations to the full sample. We note that the obtained SFR[O II] flux relation after calibrations is almost identical to the classical relation given by Kennicutt (1998). In principle, the uncertainty of this calibration, which is found to be $50 \%$ based on current data, can be improved by carrying out additional photometric and spectroscopic observations of the lensed sources.

When deriving SNIa rates, the SFH of each individual lensed sources is assumed to follow the cosmic star formation density evolution trend given in Madau \& Dickinson (2014) up to an overall normalization factor. In practice, however, the SFH for each individual galaxy will not follow this cosmic evolution trend exactly, since it only represents a global average across all galaxies. We therefore investigate the uncertainty introduced to the inferred SNIa rate by the choice of SFH by computing the SNIa rate under the assumption of a constant SFR until the point of observation for each lensed source. Compared to the SNIa rates reported above, the alternative SNIa rates vary from $-20 \%$ to $+3 \%$, with a median relative difference of $-10 \%$ across all lensed sources. This level of variation is much smaller than the systematic uncertainty in the SNIa rate of $\sim 50 \%$. The integrated change in the total SNIa rate of the entire lens compilation is relatively small $-4 \%$. We find that the SNIa rate is primarily determined by the most recent SFH because of the rapid decline in the delay-time function. In both SFH scenarios considered, the cumulative SNIa rates have already reached $50 \%$ and $90 \%$ within the recent 0.03 and $2 \mathrm{Gyr}$. In fact, the SNIa rate scales almost linearly with the observed SFR with a slope of $4.5 \times 10^{-4} \mathrm{SNe}$ $\mathrm{yr}^{-1}\left(M_{\odot} \mathrm{yr}^{-1}\right)^{-1}$, consistent with previous findings (e.g., Sullivan et al. 2006; Smith et al. 2012; Graur et al. 2015). Overall, this test suggests that different choices of the SFH would only introduce a small uncertainty in the SNIa rate, especially in the aggregate across the entire lens compilation.

Compared to a blind search, our proposed targeted search is significantly more efficient in finding strongly lensed SN events, especially ones with wide image separations. For example, 130 strongly lensed SN events with image separations larger than 0 ". 5 will be detected by LSST after a 10-year blind search of $\sim 20,000$ square degrees' sky (Oguri \& Marshall 2010). Considering the $\sim 10$ square degree field of view of LSST, at least 2000 frames need to be monitored to yield these SN detections. The detection efficiency, defined as the yield of strongly lensed $\mathrm{SN}$ events per exposure time, of this blind search is thus $\sim 0.006$ event per year per frame. Our proposed Program 2 strategy, with the same depth as LSST but targeted at known strong lenses, has a detection efficiency of $\sim 0.025$ event per year per frame, and the expected median image separation is $2^{\prime \prime}$. Furthermore, none of the previous predictions for the number of strongly lensed SNe in LSST considered the effect of dust extinction, which is known to significantly reduce the number of detections. (Our detectable $\mathrm{SN}$ rates and detection efficiencies would increase by a factor of $\sim 1.5$ at LSST depth if the applied dust extinctions were removed.) Thousands of new strong-lens systems will be discovered in the near future from dedicated surveys (e.g., Marshall et al. 2009; Oguri \& Marshall 2010; Collett 2015; McKean et al. 2015; Diehl et al. 2017). The application of an efficient strategy such as ours to the monitoring of these new lenses will significantly increase the yield of strongly lensed SNe.

A sample of strongly lensed SNe, especially SNIa, will be a powerful resource for cosmological studies. Time-delay cosmography with lensed QSOs is limited by the microlensing effect, which causes uncorrelated variability in the fluxes of different lensed images in an unpredictable manner and therefore complicates the time-delay measurement (e.g., Blandford \& Narayan 1992; Wyithe \& Turner 2002; Dobler \& Keeton 2006; Morgan et al. 2012; Tewes et al. 2013; More et al. 2017; Yahalomi et al. 2017; Tie \& Kochanek 2018). Compared to QSOs, for which light curves are typically stochastic with variability timescales extending to years, SNe have single-peak, more homogeneous, and standardizable light curves that develop on timescales of weeks. It is therefore much easier to determine time delays for lensed $\mathrm{SNe}$ than lensed QSOs in the presence of the microlensing effect. Furthermore, Goldstein et al. (2018) recently found that accurate time-delay measurements can be obtained from early time color curves of strongly lensed SNIa, which are suggested to be almost immune to the microlensing effect. Multiply lensed SN images increase the effective number of SN events for observational study. In addition, with the aid of lensing magnification, more $\mathrm{SNe}$ at higher redshifts with better measured light curves can be obtained, which can enable a more extensive study of the expansion history of the universe at earlier times. 
The standard-candle nature of $\mathrm{SNe}$ also provides extra constraining power on the mass distribution of the lens galaxy, which holds important clues to galaxy formation and evolution (e.g., Gnedin et al. 2004; Nipoti et al. 2004; Gustafsson et al. 2006; Romano-Díaz et al. 2008; Abadi et al. 2010; Duffy et al. 2010; Governato et al. 2010; Bolton et al. 2012a; Martizzi et al. 2012; Dubois et al. 2013; Velliscig et al. 2014; Shu et al. 2015). More accurate lens-galaxy mass distributions could be obtained when the mass-sheet and source-position transformation degeneracies are broken with known lensing magnifications. However, absolute lensing magnifications are not direct observables but rather are degenerate with the intrinsic luminosities of the lensed source, which in most cases are unknown. Strongly lensed $\mathrm{SNe}$ with intrinsic luminosities that can be derived from lensing-independent observables are an important exception, and hence can provide better constraints on the lens-galaxy mass distributions (e.g., Witt et al. 1995; Bolton \& Burles 2003; Oguri \& Kawano 2003; Mörtsell et al. 2005).

Multiply imaged SNe can furthermore revolutionize the observational study of SN early and pre-explosion phases, which are driven by simple, well understood physics and thus provide crucial constraints on the progenitor properties and SN explosion mechanisms. For instance, Pritchard et al. (2014) and Ganot et al. (2016) highlight the importance of early UV emissions in CCSNe in determining the physical properties of the progenitors. Tornambé et al. (2018) suggest that X-ray features in advance of SNIa explosions are determined by the rotation of the progenitors, which can be used to distinguish between different progenitor scenarios. Such types of observations are currently limited to a handful of unlensed $\mathrm{SNe}$ for which archival data or prompt follow-up observations are available (e.g., Gal-Yam et al. 2011; Li et al. 2011a; Nugent et al. 2011; Dilday et al. 2012; Cao et al. 2013; Gal-Yam et al. 2014; Kelly et al. 2014; McCully et al. 2014). The delays in the arrival times of multiple SN images, typically on scales from 1 day to 100 days, essentially replay the $\mathrm{SN}$ explosions multiple times. The approximate explosion times and exact locations can be predicted from the lens model after the arrival of the leading SN image. Intensive real-time monitoring of the $\mathrm{SN}$ site before its reappearance can be scheduled, which will greatly expand the possibilities for early and pre-explosion SN observations.

\section{Conclusions}

In this paper, we propose a highly efficient strategy of finding strongly lensed SNe by monitoring known galaxy-scale strong-lens systems. We assemble a compilation of 128 galaxy-galaxy strong-lens systems from the SLACS Survey, the S4TM Survey, and the BELLS Survey. All the lensed sources in this lens compilation are galaxies at redshifts of $0.2-1.5$ with significant recent star formation. The median lensed image separation in the sample is $2^{\prime \prime}$. The main findings based on this particular lens compilation are as follows.

1. Assuming a Salpeter IMF, the intrinsic SFRs of the lensed sources determined from the observed [O II] fluxes range from $0.3 M_{\odot} \mathrm{yr}^{-1}$ to $267 M_{\odot} \mathrm{yr}^{-1}$. The lensing magnification and fiber loss are corrected based on lens models from the HST imaging data. The median SFRs for the SLACS, S4TM, and BELLS lensed sources are $6 M_{\odot} \mathrm{yr}^{-1}, 5 M_{\odot} \mathrm{yr}^{-1}$, and $68 M_{\odot} \mathrm{yr}^{-1}$, respectively. We find that the SFRs become higher at higher redshifts, which is primarily determined by the selection effect that sources at higher redshifts are biased to have higher intrinsic [O II] flux given a fixed observer-frame detection limit.

2. The expected overall occurrence rates of SNIa and CCSN in the lensed sources are estimated either directly from the SFRs (for CCSN) or by convolving fiducial SFHs with an SNIa delay-time distribution. For individual lensed sources, the SNIa rates are in the range of 0.0002-0.09 events per year, and the CCSN rates are in the range of $0.002-0.8$ events per year. On average, the SNIa rate is a factor of $\sim 8$ lower than the CCSN rate. For the full lens compilation, we predict $1.23 \pm 0.12$ strongly lensed SNIa events per year, and $10.4 \pm 1.1$ strongly lensed CCSN events per year. Mainly due to the same selection effect, the $\mathrm{SN}$ rates are higher at progressively higher redshifts.

3. We quantify the expected occurrence rates of detectable strongly lensed SNIa and CCSN events in two nominal monitoring programs with different depths. We assume a conservative fiducial magnification factor of 5 for the most magnified SN image. Within this lens compilation, our Program 1 is expected to detect approximately 0.003 strongly lensed SNIa event and 0.019 strongly lensed CCSN event per year, while our Program2 can detect 0.49 strongly lensed SNIa event and 2.1 strongly lensed CCSN events per year. This detection efficiency is significantly higher than that of a blind search.

4. For this lens compilation, it is more efficient to target either higher-redshift or lower-redshift strong-lens systems depending on whether the single-visit $r$-band depth is brighter than $24.5 \mathrm{mag}$ or not.

In closing, we emphasize that the fundamental principle of our proposed strategy is to focus on small targeted sky areaswhere known strong-lens systems are located-as being more favorable sites for finding strongly lensed SNe. In the era that hundreds of new SNe (including strongly lensed SNe) are expected to be discovered every night by high-cadence, all-sky surveys such as ZTF and LSST, our proposed strategy simply helps in prompt and efficient identifications of the stronglensing nature of the detected SNe. Meanwhile, it allows telescopes with small fields of view and limited observing time to efficiently discover strongly lensed $\mathrm{SNe}$ with a pencil-beam monitoring strategy. As we show in the paper, such targetedsearch programs with depths comparable to that of LSST can be productive today, which is particularly worthwhile considering that LSST is scheduled to start operation in 2022 (LSST Science Collaboration et al. 2017).

We thank the anonymous referee for comments that substantially improved the manuscript. We are grateful for the helpful discussions with Matt Auger, Peter Brown, Kyle Dawson, Mark Dickinson, Thomas Matheson, Gautham Narayan, Justin Roberts-Pierel, Steven Rodney, Hanna Sai, Lou Strolger, and Danfeng Xiang. Y.S. has been supported by the National Natural Science Foundation of China (NSFC; No. 11603032 and 11333008), the 973 program (No. 2015CB857003), and the Royal Society-K. C. Wong International Fellowship (NF170995). S.M. acknowledges support from the NSFC (grant No. 11333003, 11390372). G.L. is supported by the NSFC (No.11673065, 11273061). 


\section{References}

Abadi, M. G., Navarro, J. F., Fardal, M., Babul, A., \& Steinmetz, M. 2010, MNRAS, 407, 435

Adams, S. M., Kochanek, C. S., Beacom, J. F., Vagins, M. R., \& Stanek, K. Z. 2013, ApJ, 778, 164

Alam, S., Ata, M., Bailey, S., et al. 2017, MNRAS, 470, 2617

Anderson, L., Aubourg, É., Bailey, S., et al. 2014, MNRAS, 441, 24

Argence, B., \& Lamareille, F. 2009, A\&A, 495, 759

Auger, M. W., Treu, T., Gavazzi, R., et al. 2010, ApJL, 721, L163

Barbary, K. 2014, sncosmo, v0.4.2, Zenodo, doi:10.5281/zenodo.11938

Barbon, R., Ciatti, F., \& Rosino, L. 1979, A\&A, 72, 287

Bassett, B., Becker, A., Brewington, H., et al. 2006, CBET, 667, 1

Bellm, E. 2014, in The Third Hot-wiring the Transient Universe Workshop, ed.

P. R. Wozniak et al., 27

Bennett, C. L., Larson, D., Weiland, J. L., et al. 2013, ApJS, 208, 20

Betoule, M., Kessler, R., Guy, J., et al. 2014, A\&A, 568, A22

Blandford, R. D., \& Narayan, R. 1992, ARA\&A, 30, 311

Bolton, A. S., Brownstein, J. R., Kochanek, C. S., et al. 2012a, ApJ, 757, 82

Bolton, A. S., \& Burles, S. 2003, ApJ, 592, 17

Bolton, A. S., Burles, S., Koopmans, L. V. E., et al. 2008, ApJ, 682, 964

Bolton, A. S., Burles, S., Koopmans, L. V. E., Treu, T., \& Moustakas, L. A. 2006a, ApJ, 638, 703

Bolton, A. S., Burles, S., Schlegel, D. J., Eisenstein, D. J., \& Brinkmann, J. 2004, AJ, 127, 1860

Bolton, A. S., Moustakas, L. A., Stern, D., et al. 2006b, ApJL, 646, L45

Bolton, A. S., Schlegel, D. J., Aubourg, É., et al. 2012b, AJ, 144, 144

Bonvin, V., Courbin, F., Suyu, S. H., et al. 2017, MNRAS, 465, 4914

Brewer, B. J., Marshall, P. J., Auger, M. W., et al. 2014, MNRAS, 437, 1950

Brinchmann, J., Charlot, S., White, S. D. M., et al. 2004, MNRAS, 351, 1151

Browne, I. W. A., Wilkinson, P. N., Jackson, N. J. F., et al. 2003, MNRAS, 341,13

Brownstein, J. R., Bolton, A. S., Schlegel, D. J., et al. 2012, ApJ, 744, 41

Bussmann, R. S., Pérez-Fournon, I., Amber, S., et al. 2013, ApJ, 779, 25

Calzetti, D., Armus, L., Bohlin, R. C., et al. 2000, ApJ, 533, 682

Cao, Y., Kasliwal, M. M., Arcavi, I., et al. 2013, ApJL, 775, L7

Cappellari, M., McDermid, R. M., Alatalo, K., et al. 2012, Natur, 484, 485

Cardiel, N., Elbaz, D., Schiavon, R. P., et al. 2003, ApJ, 584, 76

Christensen, L., Richard, J., Hjorth, J., et al. 2012, MNRAS, 427, 1953

Collett, T. E. 2015, ApJ, 811, 20

Conroy, C., Dutton, A. A., Graves, G. J., Mendel, J. T., \& van Dokkum, P. G. 2013, ApJL, 776, L26

Dahlén, T., \& Fransson, C. 1999, A\&A, 350, 349

Dahlen, T., Strolger, L.-G., Riess, A. G., et al. 2004, ApJ, 613, 189

Dahlen, T., Strolger, L.-G., Riess, A. G., et al. 2012, ApJ, 757, 70

de Jaeger, T., González-Gaitán, S., Hamuy, M., et al. 2017, ApJ, 835, 166

Diehl, H. T., Buckley-Geer, E. J., Lindgren, K. A., et al. 2017, ApJS, 232, 15

Diehl, R., Halloin, H., Kretschmer, K., et al. 2006, Natur, 439, 45

Dilday, B., Howell, D. A., Cenko, S. B., et al. 2012, Sci, 337, 942

Dobler, G., \& Keeton, C. R. 2006, ApJ, 653, 1391

Doggett, J. B., \& Branch, D. 1985, AJ, 90, 2303

Doi, M., Tanaka, M., Fukugita, M., et al. 2010, AJ, 139, 1628

Dubois, Y., Gavazzi, R., Peirani, S., \& Silk, J. 2013, MNRAS, 433, 3297

Duffy, A. R., Schaye, J., Kay, S. T., et al. 2010, MNRAS, 405, 2161

Eisenstein, D. J., Zehavi, I., Hogg, D. W., et al. 2005, ApJ, 633, 560

Falco, E. E., Gorenstein, M. V., \& Shapiro, I. I. 1985, ApJL, 289, L1

Faure, C., Kneib, J.-P., Covone, G., et al. 2008, ApJS, 176, 19

Ferreras, I., la Barbera, F., de la Rosa, I. G., et al. 2013, MNRAS, 429, L15

Firth, R. E., Sullivan, M., Gal-Yam, A., et al. 2015, MNRAS, 446, 3895

Foley, R. J., van Dyk, S. D., Jha, S. W., et al. 2015, ApJL, 798, L37

Foxley-Marrable, M., Collett, T. E., Vernardos, G., Goldstein, D. A., \& Bacon, D. 2018, MNRAS, 478, 5081

Gallagher, J. S., Hunter, D. A., \& Bushouse, H. 1989, AJ, 97, 700

Gal-Yam, A., Arcavi, I., Ofek, E. O., et al. 2014, Natur, 509, 471

Gal-Yam, A., Kasliwal, M. M., Arcavi, I., et al. 2011, ApJ, 736, 159

Ganot, N., Gal-Yam, A., Ofek, E. O., et al. 2016, ApJ, 820, 57

Gilbank, D. G., Baldry, I. K., Balogh, M. L., Glazebrook, K., \& Bower, R. G. 2010, MNRAS, 405, 2594

Gnedin, O. Y., Kravtsov, A. V., Klypin, A. A., \& Nagai, D. 2004, ApJ, 616, 16 Goldstein, D. A., \& Nugent, P. E. 2017, ApJL, 834, L5

Goldstein, D. A., Nugent, P. E., Kasen, D. N., \& Collett, T. E. 2018, ApJ, 855,22

Goobar, A., Amanullah, R., Kulkarni, S. R., et al. 2017, Sci, 356, 291

Goobar, A., Paech, K., Stanishev, V., et al. 2009, A\&A, 507, 71

Gorenstein, M. V., Shapiro, I. I., \& Falco, E. E. 1988, ApJ, 327, 693

Governato, F., Brook, C., Mayer, L., et al. 2010, Natur, 463, 203
Graur, O., Bianco, F. B., \& Modjaz, M. 2015, MNRAS, 450, 905

Greggio, L., Renzini, A., \& Daddi, E. 2008, MNRAS, 388, 829

Gustafsson, M., Fairbairn, M., \& Sommer-Larsen, J. 2006, PhRvD, 74, 123522

Hayashi, M., Sobral, D., Best, P. N., Smail, I., \& Kodama, T. 2013, MNRAS, 430, 1042

He, S., Wang, L., \& Huang, J. Z. 2018, ApJ, 857, 110

Hicks, E. K. S., Malkan, M. A., Teplitz, H. I., McCarthy, P. J., \& Yan, L. 2002, ApJ, 581, 205

Hoeflich, P., Hsiao, E. Y., Ashall, C., et al. 2017, ApJ, 846, 58

Hogg, D. W., Cohen, J. G., Blandford, R., \& Pahre, M. A. 1998, ApJ, 504, 622 Hopkins, A. M., \& Beacom, J. F. 2006, ApJ, 651, 142

Hopkins, A. M., Miller, C. J., Nichol, R. C., et al. 2003, ApJ, 599, 971

Hsiao, E. Y., Conley, A., Howell, D. A., et al. 2007, ApJ, 663, 1187

Impey, C. D., Falco, E. E., Kochanek, C. S., et al. 1998, ApJ, 509, 551

Inada, N., Oguri, M., Shin, M.-S., et al. 2012, AJ, 143, 119

Ivezic, Z., Tyson, J. A., Abel, B., et al. 2008, arXiv:0805.2366

Jansen, R. A., Franx, M., \& Fabricant, D. 2001, ApJ, 551, 825

Jones, M. I., Hamuy, M., Lira, P., et al. 2009, ApJ, 696, 1176

Joubert, N., Li, W., Itagaki, K., \& Nakano, S. 2006, CBET, 609, 1

Karman, W., Grillo, C., Balestra, I., et al. 2016, A\&A, 585, A27

Kasen, D., \& Woosley, S. E. 2009, ApJ, 703, 2205

Kelly, P. L., Fox, O. D., Filippenko, A. V., et al. 2014, ApJ, 790, 3

Kelly, P. L., Rodney, S. A., Treu, T., et al. 2015, Sci, 347, 1123

Kelly, P. L., Rodney, S. A., \& Treu, T. , 2016, ApJL, 819, L8

Kennicutt, R. C., Jr. 1983, ApJ, 272, 54

Kennicutt, R. C., Jr. 1992, ApJ, 388, 310

Kennicutt, R. C., Jr. 1998, ARA\&A, 36, 189

Kewley, L. J., Geller, M. J., \& Jansen, R. A. 2004, AJ, 127, 2002

Koopmans, L. V. E., Treu, T., Fassnacht, C. D., Blandford, R. D., \& Surpi, G. 2003, ApJ, 599, 70

Kostrzewa-Rutkowska, Z., Wyrzykowski, Ł., \& Jaroszyński, M. 2013, MNRAS, 429, 2392

Kroupa, P. 2001, MNRAS, 322, 231

la Barbera, F., Ferreras, I., Vazdekis, A., et al. 2013, MNRAS, 433, 3017

Li, H., Ge, J., Mao, S., et al. 2017, ApJ, 838, 77

Li, W., Bloom, J. S., Podsiadlowski, P., et al. 2011a, Natur, 480, 348

Li, W., Chornock, R., Leaman, J., et al. 2011b, MNRAS, 412, 1473

Li, W., Leaman, J., Chornock, R., et al. 2011c, MNRAS, 412, 1441

Liesenborgs, J., \& de Rijcke, S. 2012, MNRAS, 425, 1772

LSST Science Collaboration, Marshall, P., Anguita, T., et al. 2017, arXiv:1708. 04058

Madau, P., della Valle, M., \& Panagia, N. 1998, MNRAS, 297, L17

Madau, P., \& Dickinson, M. 2014, ARA\&A, 52, 415

Mannucci, F., Maiolino, R., Cresci, G., et al. 2003, A\&A, 401, 519

Maoz, D., Mannucci, F., \& Brandt, T. D. 2012, MNRAS, 426, 3282

Maoz, D., Mannucci, F., Li, W., et al. 2011, MNRAS, 412, 1508

Markwardt, C. B. 2009, in ASP Conf. Ser. 411, Astronomical Data Analysis Software and Systems XVIII, ed. D. A. Bohlender, D. Durand, \& P. Dowler (San Francisco, CA: ASP), 251

Marshall, P. J., Hogg, D. W., Moustakas, L. A., et al. 2009, ApJ, 694, 924

Martizzi, D., Teyssier, R., Moore, B., \& Wentz, T. 2012, MNRAS, 422, 3081

McCully, C., Jha, S. W., Foley, R. J., et al. 2014, Natur, 512, 54

McKean, J., Jackson, N., Vegetti, S., et al. 2015, in Advancing Astrophysics with the Square Kilometre Array (AASKA14) (Trieste: SISSA), 84

Melinder, J., Dahlen, T., Mencía Trinchant, L., et al. 2012, A\&A, 545, A96

More, A., Cabanac, R., More, S., et al. 2012, ApJ, 749, 38

More, A., Suyu, S. H., Oguri, M., More, S., \& Lee, C.-H. 2017, ApJL, 835, L25

More, A., Verma, A., Marshall, P. J., et al. 2016, MNRAS, 455, 1191

Morgan, C. W., Hainline, L. J., Chen, B., et al. 2012, ApJ, 756, 52

Morozova, V., Piro, A. L., \& Valenti, S. 2017, ApJ, 838, 28

Mörtsell, E., Dahle, H., \& Hannestad, S. 2005, ApJ, 619, 733

Mostek, N., Coil, A. L., Moustakas, J., Salim, S., \& Weiner, B. J. 2012, ApJ, 746,124

Moustakas, J., Kennicutt, R. C., Jr., \& Tremonti, C. A. 2006, ApJ, 642, 775

Muñoz, J. A., Falco, E. E., Kochanek, C. S., et al. 1998, Ap\&SS, 263, 51

Muzzin, A., Labbé, I., Franx, M., et al. 2012, ApJ, 761, 142

Negrello, M., Amber, S., Amvrosiadis, A., et al. 2017, MNRAS, 465, 3558

Nipoti, C., Treu, T., Ciotti, L., \& Stiavelli, M. 2004, MNRAS, 355, 1119

Nugent, P., Sullivan, M., Ellis, R., et al. 2006, ApJ, 645, 841

Nugent, P. E., Sullivan, M., Cenko, S. B., et al. 2011, Natur, 480, 344

Oguri, M., \& Kawano, Y. 2003, MNRAS, 338, L25

Oguri, M., \& Marshall, P. J. 2010, MNRAS, 405, 2579

Olivares, E. F., Hamuy, M., Pignata, G., et al. 2010, ApJ, 715, 833

Pan, T., \& Loeb, A. 2013, MNRAS, 435, L33 
Pawase, R. S., Courbin, F., Faure, C., Kokotanekova, R., \& Meylan, G. 2014, MNRAS, 439, 3392

Percival, W. J., Reid, B. A., Eisenstein, D. J., et al. 2010, MNRAS, 401, 2148 Perlmutter, S., Aldering, G., Goldhaber, G., et al. 1999, ApJ, 517, 565

Petrushevska, T., Amanullah, R., Goobar, A., et al. 2016, A\&A, 594, A54

Petrushevska, T., Goobar, A., Lagattuta, D. J., et al. 2018, A\&A, 614, A103

Planck Collaboration, Ade, P. A. R., Aghanim, N., et al. 2016, A\&A, 594, A13

Postman, M., Coe, D., Benitez, N., et al. 2012, ApJS, 199, 25

Poznanski, D., Butler, N., Filippenko, A. V., et al. 2009, ApJ, 694, 1067

Pritchard, T. A., Roming, P. W. A., Brown, P. J., Bayless, A. J., \& Frey, L. H 2014, ApJ, 787, 157

Pugh, H., Park, S., \& Li, W. 2004, IAUC, 8425, 1

Quider, A. M., Pettini, M., Shapley, A. E., \& Steidel, C. C. 2009, MNRAS, 398, 1263

Quimby, R. M., Oguri, M., More, A., et al. 2014, Sci, 344, 396

Richardson, D., Branch, D., Casebeer, D., et al. 2002, AJ, 123, 745

Richardson, D., Jenkins, R. L., III, Wright, J., \& Maddox, L. 2014, AJ, 147,118

Richmond, M. W., Filippenko, A. V., \& Galisky, J. 1998, PASP, 110, 553

Riess, A. G., Filippenko, A. V., Challis, P., et al. 1998, AJ, 116, 1009

Rodney, S. A., Patel, B., Scolnic, D., et al. 2015, ApJ, 811, 70

Romano-Díaz, E., Shlosman, I., Hoffman, Y., \& Heller, C. 2008, ApJL, 685, L105

Rubin, D., \& Hayden, B. 2016, ApJL, 833, L30

Saha, P. 2000, AJ, 120, 1654

Sako, M., Bassett, B., Becker, A. C., et al. 2018, PASP, 130, 064002

Salpeter, E. E. 1955, ApJ, 121, 161

Sanders, N. E., Soderberg, A. M., Gezari, S., et al. 2015, ApJ, 799, 208

Schmidt, B. P., Suntzeff, N. B., Phillips, M. M., et al. 1998, ApJ, 507, 46

Schneider, P., \& Sluse, D. 2013, A\&A, 559, A37

Schneider, P., \& Sluse, D. 2014, A\&A, 564, A103

Shu, Y., Bolton, A. S., Brownstein, J. R., et al. 2015, ApJ, 803, 71

Shu, Y., Bolton, A. S., Kochanek, C. S., et al. 2016a, ApJ, 824, 86

Shu, Y., Bolton, A. S., Mao, S., et al. 2016b, ApJ, 833, 264

Shu, Y., Bolton, A. S., Moustakas, L. A., et al. 2016c, ApJ, 820, 43

Shu, Y., Brownstein, J. R., Bolton, A. S., et al. 2017, ApJ, 851, 48

Smith, M., Nichol, R. C., Dilday, B., et al. 2012, ApJ, 755, 61

Sobral, D., Best, P. N., Matsuda, Y., et al. 2012, MNRAS, 420, 1926

Soderberg, A. M., Berger, E., Page, K. L., et al. 2008, Natur, 453, 469

Sonnenfeld, A., Chan, J. H. H., Shu, Y., et al. 2018, PASJ, 70, S29

Sonnenfeld, A., Gavazzi, R., Suyu, S. H., Treu, T., \& Marshall, P. J. 2013, ApJ, 777, 97
Sonnenfeld, A., Treu, T., Gavazzi, R., et al. 2012, ApJ, 752, 163 Spilker, J. S., Marrone, D. P., Aravena, M., et al. 2016, ApJ, 826, 112

Spiniello, C., Trager, S., Koopmans, L. V. E., \& Conroy, C. 2014, MNRAS, 438, 1483

Spiniello, C., Trager, S. C., Koopmans, L. V. E., \& Chen, Y. P. 2012, ApJL, 753, L32

Stark, D. P., Auger, M., Belokurov, V., et al. 2013, MNRAS, 436, 1040

Stark, D. P., Walth, G., Charlot, S., et al. 2015, MNRAS, 454, 1393

Strader, J., Caldwell, N., \& Seth, A. C. 2011, AJ, 142, 8

Strolger, L.-G., Dahlen, T., Rodney, S. A., et al. 2015, ApJ, 813, 93

Sullivan, M., le Borgne, D., Pritchet, C. J., et al. 2006, ApJ, 648, 868

Suyu, S. H., Auger, M. W., Hilbert, S., et al. 2013, ApJ, 766, 70

Suyu, S. H., Marshall, P. J., Auger, M. W., et al. 2010, ApJ, 711, 201

Suyu, S. H., Treu, T., Hilbert, S., et al. 2014, ApJL, 788, L35

Taddia, F., Stritzinger, M. D., Bersten, M., et al. 2018, A\&A, 609, A136

Talia, M., Cimatti, A., Pozzetti, L., et al. 2015, A\&A, 582, A80

Tammann, G. A., Loeffler, W., \& Schroeder, A. 1994, ApJS, 92, 487

Tewes, M., Courbin, F., \& Meylan, G. 2013, A\&A, 553, A120

Tie, S. S., \& Kochanek, C. S. 2018, MNRAS, 473, 80

Tornambé, A., Piersanti, L., Raimondo, G., \& Delgrande, R. 2018, MNRAS, 475, 3076

Tortora, C., Romanowsky, A. J., \& Napolitano, N. R. 2013, ApJ, 765, 8

Treu, T., Auger, M. W., Koopmans, L. V. E., et al. 2010, ApJ, 709, 1195

Treu, T., Dutton, A. A., Auger, M. W., et al. 2011, MNRAS, 417, 1601

Treu, T., \& Koopmans, L. V. E. 2002, MNRAS, 337, L6

Tsujimoto, T., Yoshii, Y., Nomoto, K., et al. 1997, ApJ, 483, 228

van Dokkum, P. G., \& Conroy, C. 2010, Natur, 468, 940

Velliscig, M., van Daalen, M. P., Schaye, J., et al. 2014, MNRAS, 442, 2641

Vieira, J. D., Marrone, D. P., Chapman, S. C., et al. 2013, Natur, 495, 344

Walsh, D., Carswell, R. F., \& Weymann, R. J. 1979, Natur, 279, 381

Wang, X., Wang, L., Filippenko, A. V., Zhang, T., \& Zhao, X. 2013, Sci, 340,170

Wang, Y., Zhao, G.-B., Chuang, C.-H., et al. 2017, MNRAS, 469, 3762

Witt, H. J., Mao, S., \& Keeton, C. R. 2000, ApJ, 544, 98

Witt, H. J., Mao, S., \& Schechter, P. L. 1995, ApJ, 443, 18

Wong, K. C., Suyu, S. H., Auger, M. W., et al. 2017, MNRAS, 465, 4895

Wucknitz, O. 2002, MNRAS, 332, 951

Wuyts, S., Förster Schreiber, N. M., Genzel, R., et al. 2012, ApJ, 753, 114

Wyithe, J. S. B., \& Turner, E. L. 2002, ApJ, 575, 650

Yahalomi, D. A., Schechter, P. L., \& Wambsganss, J. 2017, arXiv:1711.07919

Zhao, G.-B., Wang, Y., Saito, S., et al. 2017, MNRAS, 466, 762 